\title{
Parasite Mitogen-Activated Protein Kinases as Drug Discovery Targets to Treat Human Protozoan Pathogens
}

\author{
Michael J. Brumlik, Srilakshmi Pandeswara, Sara M. Ludwig, Kruthi Murthy, \\ and Tyler J. Curiel
}

Department of Medicine, School of Medicine, and Program in Immunology and Microbiology, Graduate School of Biomedical Sciences, University of Texas Health Science Center, San Antonio, TX 78229, USA

Correspondence should be addressed to Michael J. Brumlik, brumlik@uthscsa.edu and Tyler J. Curiel, curielt@uthscsa.edu

Received 16 August 2010; Revised 12 November 2010; Accepted 7 December 2010

Academic Editor: M. Gaestel

Copyright (๑ 2011 Michael J. Brumlik et al. This is an open access article distributed under the Creative Commons Attribution License, which permits unrestricted use, distribution, and reproduction in any medium, provided the original work is properly cited.

Protozoan pathogens are a highly diverse group of unicellular organisms, several of which are significant human pathogens. One group of protozoan pathogens includes obligate intracellular parasites such as agents of malaria, leishmaniasis, babesiosis, and toxoplasmosis. The other group includes extracellular pathogens such as agents of giardiasis and amebiasis. An unfortunate unifying theme for most human protozoan pathogens is that highly effective treatments for them are generally lacking. We will review targeting protozoan mitogen-activated protein kinases (MAPKs) as a novel drug discovery approach towards developing better therapies, focusing on Plasmodia, Leishmania, and Toxoplasma, about which the most is known.

\section{General Properties of MAPKs}

Virtually all eukaryotic organisms possess MAPKs, signal transduction molecules that regulate cell functions such as tissue morphogenesis, cytoskeletal rearrangements, proliferation, differentiation, survival, immune responses, and adaptation/stress-response [1-3]. Encephalitozoon cuniculi is the only example to date of a eukaryote apparently lacking any MAPKs [4]. The MAPK superfamily, which evolved 1.0 to 1.5 billion years ago [5], comprises proline-directed serine/threonine kinases that are classified based on the primary amino acid sequence within the catalytic domains and must possess a [TS] XX[LIVM]XT[RK] [WY]YRXPEX[LIVM] signature sequence at its core [6-8]. The phosphorylation lip (solid underline beneath the sequence above) is required for MAPK activation by upstream regulators and is contiguous with the proline-directed $(\mathrm{P}+1)$ peptide binding pocket (double underline beneath the sequence above), conferring substrate specificity, and is capable of being singly $[(\mathrm{pT}) \mathrm{XX})]$ or dually $[(\mathrm{pT}) \mathrm{X}(\mathrm{pY})]$ phosphorylated in response to particular extracellular stimuli [9]. In addition,
MAPKs possess 11 subdomains $[5,10]$ with numerous highly conserved residues required for ATP binding, phosphotransferase activity, and substrate specificity [7].

MAPKs are often controlled by highly evolutionarily conserved regulatory cascades involving sequential phosphorylation by three component modules consisting of MAPK kinase kinases (MKKKs, Ste11-like kinases) and MAPK kinases (MKKs, Ste7-like kinases), terminating in the phosphorylation of specific MAPKs [11]. Many MAPK cascades have recently been expanded to include a fourth tier involving proteins aptly termed MKKKKs (Ste20-like kinases) [12] that can either serve in a noncatalytic capacity as a scaffold to promote pathway assembly (and MKKK autoactivation) or can phosphorylate specific MKKKs [13]. Once activated, MAPKs phosphorylate a wide variety of proteins including MAPK-activated protein kinases and transcription factors, ultimately resulting in changes in gene expression $[14,15]$. MAPK signaling can also have additional epigenetic effects by affecting histone modification [16].

MAPKs are grouped into subfamilies on the basis of amino acid sequence similarity, mechanism of activation, 
and the type of MAPK cascade to which they belong. Cyclindependent kinases share very high amino acid sequence identity with MAPKs [17] but generally lack a phosphorylation lip. Differences in the precise amino acid composition of the phosphorylation lip have historically been used to classify MAPKs, as outlined below. Our phylogenetic studies [18] have established, however, that homology between many short strings of amino acids found in MAPKs is of equal or greater importance when classifying MAPKs within different subfamilies.

Four conventional MAPK subfamilies exist, which are also described as "typical", that is, capable of dual phosphorylation [8]. These conventional MAPK groups include the extracellular signal-regulated kinases (e.g., mammalian ERK1 and ERK2, possessing a TEY motif at the phosphorylation lip, [19]), c-Jun-activated kinases (e.g., mammalian JNK1, JNK2, and JNK3 (TPY motif) [20]), p38 stressresponse MAPKs (e.g., mammalian p38 $\alpha$, p38 $\beta$, p38 $\gamma$, and p38 $\delta$ (TGY motif), [21]), and mammalian ERK5 (big MAPK-1, BMK-1 (TEY motif) [22, 23]). ERK5 is unusual because it possesses a long carboxy-terminal extension consisting of a transactivation domain and a nuclear localization signal facilitating translocation into the nucleus upon MAPK activation [8]. Multiple isoforms of MAPKs often exist within individual cells, which can either be activated by different MKKs or can themselves phosphorylate alternate downstream substrates [24]. Additional phylogenetically distinct MAPK subfamilies are defined by categorizing distantly related MAPKs including those from plants (TEY motif), yeasts (T[EN]Y motif), and protozoans (TXY motif, where $\mathrm{X}$ is often $\mathrm{D}$ or $\mathrm{E}$, but many exceptions exist) $[5,18]$.

Several atypical MAPK subfamilies also exist, largely representing MAPKs that can only be monophosphorylated within their activation loops. Mammalian ERK3 [25] and ERK4 [8], possessing an SEG motif in the phosphorylation lip and an RXPR motif in the substrate binding pocket, are representative members of one major subfamily of atypical MAPKs, while Nemo-like kinases (NLKs, with a T[HQ]E motif) comprise a second major atypical MAPK subfamily [26]. Greater sequence diversity exists in the phosphorylation lip of atypical protozoan MAPKs (most commonly TGH or TSH motifs) compared to metazoan MAPKs, but members of this subfamily otherwise closely resemble typical MAPKs.

Monophosphorylated human ERK2 has 10- to 100-fold less kinase activity than dually phosphorylated ERK2 [27], illustrating that dual phosphorylation (as is the case for typical MAPKs) achieves greater signal amplification and range of responses than can be achieved by monophosphorylation (as is the case for atypical MAPKs). In addition, different upstream activators can preferentially phosphorylate the threonine or tyrosine within the activation loop of typical MAPKs, allowing signals from two different origins to elicit a response [28]. Typical MAPKs are also subject to a tertiary level of control through the expression of phosphatases specific for either phosphothreonine or phosphotyrosine in the activation loop [29].

Human ERK8 (homologous to rat ERK7) represents a prototypical member of a large atypical MAPK subfamily [30]. Although these large atypical MAPKs contain a TEY motif capable of dual phosphorylation, activation of mammalian ERK8 (or ERK7) is not under the control of any known MKK family member. Instead, they are activated by autophosphorylation of their activation loops in response to conformational changes in their carboxy-terminal extensions [31] - a highly unusual feature for mammalian MAPKs. Their carboxy-terminal extensions possess a nuclear localization signal that is only exposed in the activated state, thereby facilitating MAPK translocation to the nucleus, which in turn regulates cell proliferation [32].

We performed ClustalW alignment [33] comparing the amino acid sequences of representative metazoan (Homo sapiens [21, 34], Drosophila melanogaster [35], Caenorhabditis elegans [36]) and yeast (Saccharomyces cerevisiae [6]) p38 MAPKs to unique protozoan MAPKs described in this review (Figure 1). Human $\mathrm{p} 38 \alpha$ was selected as a prototypical MAPK for comparison for three principal reasons. First, a plethora of p38 MAPK inhibitor drugs currently exists [37, 38]. Second, the binding specificity of the pyridinylimidazole p38 MAPK inhibitor SB203580 to the ATP binding pocket of human p38 $\alpha$ is well understood $[39,40]$. Third, we have shown that p38 MAPK inhibitors effectively inhibit the in vitro replication of protozoan parasites such as Plasmodium falciparum (Brumlik et al., submitted), L. donovani (Brumlik et al., unpublished observations), and T. gondii [41]. We have further demonstrated that the pyridinylimidazole p38 MAPK inhibitor RWJ67657 protects mice from lethal challenge with T. gondii [42]. Figure 1 demonstrates that while the overall structure of MAPKs is highly conserved even between distantly related eukaryotes, unique features exist that could lead to the design of MAPK inhibitors specific for protozoan parasites.

\section{Phylum Apicomplexa}

Apicomplexa is a large, diverse phylum comprising over 5000 species, of which seven are known human pathogens (in the genera Babesia, Cryptosporidium, Cyclospora, Isospora, Plasmodium, Sarcocystis, and Toxoplasma). There are no reports of functional studies of MAPKs from Babesia, Cryptosporidium, Cyclospora, Isospora, or Sarcocystis to our knowledge. This section will thus focus on Plasmodium and Toxoplasma.

2.1. Genus Plasmodium. The genus Plasmodium contains four significant human pathogens, all agents of malaria: P. falciparum, P. vivax, P. ovale, and P. malariae. P. falciparum, which causes the most severe form of malaria, possesses only two MAPKs. Its Pfmap-1 represents a typical MAPK that is predominantly expressed in gametocytes [43] while Pfmap-2 represents an atypical MAPK (Table 1, Figure 1), which instead possesses a TSH phosphorylation lip [44]. PfPK7, which bears extremely limited homology to mammalian MKK3 and MKK6 that activate host p38 MAPK, does not appear to be a true MKK homologue. Furthermore, PfPK7 is unable to phosphorylate either recombinant Pfmap-1 or Pfmap-2 in vitro [45], suggesting that it does not represent a long-sought-after member of an MAPK cascade 


\begin{tabular}{|l|}
\hline HS_p38 $\alpha$ \\
CE_p38 \\
DM_p38 $\alpha$ \\
SC_Hog1 \\
\hline
\end{tabular}

GI_ERK1

GI_ERK2

LMa_MPK1

LMa_MPK2

LMa_MPK3

LMa_MPK4

LMa_MPK5

LMa_MPK6

LMa_MPK7

LMa_MPK8

LMa_MPK9

LMa_MPK10

LMa_MPK11

LMa_MPK12

LMa_MPK13

LMa_MPK14

LMa_MPK15

PF_Pfmap1

PF_Pfmap2

TG_TgMAPK1

TG_TgMAPK2

TG_TgMAPK3
20

30

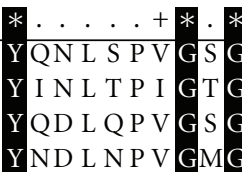

$++*$.

$+$

CA A E CTR S - G T R
40

$+.+$ KKL - S R P F Q S I I HA- - KRT Y RE KKF - NRP F Q S I I HA - - RRT Y RE

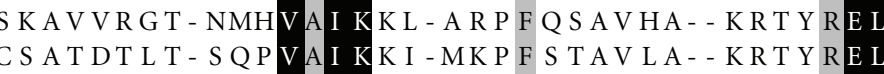
ND L N P V GMG A F G NKAVDKT T - HE TVAL KKI - F DAFQNAT DA- - QRT F RE AEAVDTRT - NTTVAI KKI - S NL F VHLVDS - - KRT L RE KV T K A L GA E I I HRVGKGAYGV R I L RH I GS WK A V NRK T - NET E I Q A Q L GQGA Y G I V T L L K I L GMGA Y G D L V KVVGF T V T S V I GHGA Y GV E T L G I L GE E V L NG I GYGAYGV Y DV L E V I GE WC A L DRR T - GK CVAL KKV - Y D A F GNVQDA WR A L ER KH - NRVVAL KK I - Y DA F QNS T DA QR T Y R E QR T F R E I A C S C L DGDTGE KVSI KKC - R DVF R DVE DG- - KRV L RE C S AVANGS - GERVAI KRL - S RVFGDLR EG- - KR I L REN CAA L DDRT - FQEVAI KRV - S RVFEDL I DG - - RR I WRE VKAR S RVT - GKLVAI KRF - - KQT EQDE HV - RKT S S RE CAAVDLR - - P FVAI KKVT - KVFDDLVDG- - RR I L RE F K CR DKR T - NR I VAV KQF - - KNF QTNA YV - RVAML RE TVMGQL GDGS FGTVSKAQNTS T- GE I VAV KKM- KQR F H SWE E C - - - LQL RE $\mathrm{Y}$ E R I I GA YQ I L GKKGEGT F S E V C S AVDAVT - NE P VA A I K RV - - - F NTVS D - - KRVLRE V AM KRVNK E I F E EV I L A - - KR I L RE T F G VAKAVS KKT - GQLVAI KKM- KQKF Y TWE E C - - VK - LPE I L VKQI GKGGFGAVEEYTDA I T - EDNVAI K T I - P S RYVN - Q E S - - RRL VRE YD I L KKVGKGAYGVVFKGRCKKN-KNIVAVKKI - F GA F QNCT DA - E I KHL I GRGSYGYVYL A Y DKNA - NKNVA I KKV - NRMF E DL I DC- VKKVGSGAYGCVA - . . . . . - KKVAVKKI - GDL F R DL I DA D I L QKL GKGAYGIVWKS T DRR T - NETVAL KKI - F DAF QNAT DA - Y E I RHL I GT GS Y GHVCE A Y DK L E - K R V

I

\section{A I KKI - L R VFEDL I DC-} $+.+$

II
60

R L L KHMK
R L L R CMC
R L L KHMD
K L L KH L R
MY L QRMD
T I L RML D
I MF L QE L D
ML L QR L R
I MF L HR L H
I DMMR F F H
E I MT S L K
L L L R I L K
RML Q L L Q
K L L R Y L Q
R V E Q L L K
I QS L R K V Q
I R L L NHF H
I K L L AHF N
K L L AY L K
I QA V R R Q
V DV V R R I H
I D I MCF L H
I I F L Y E L N
I T I L NR L K
I K I L KE L K
I MF L QE L A
I A I L NR L N
* + +. + +.
III

III

$+++++.+.++$

$+++++++++.++\ldots+++$

\begin{tabular}{|ll}
\hline HS_p38 $\alpha$ & - \\
CE_p38 & - \\
DM_p38 $\alpha$ & - \\
SC_Hog1 & - \\
EH_EhMAPK & - \\
GI_ERK1 & - \\
GI_ERK2 & - \\
LMa_MPK1 & - \\
LMa_MPK2 & - \\
LMa_MPK3 & - \\
LMa_MPK4 & - \\
LMa_MPK5 & E C \\
LMa_MPK6 & - \\
LMa_MPK7 & G \\
LMa_MPK8 & S \\
LMa_MPK9 & - \\
LMa_MPK10 & - \\
LMa_MPK11 & D \\
LMa_MPK12 & - \\
LMa_MPK13 & P \\
LMa_MPK14 & G \\
LMa_MPK15 & E A \\
PF_Pfmap1 & G \\
PF_Pfmap2 & S \\
TG_TgMAPK1 & - \\
TG_TgMAPK2 & G - \\
TG_TgMAPK3 & - \\
&
\end{tabular}

- H - E NI I DL L DV

F T P A R S

......

I T T NEN - - VND I EDVY F VSMLMGADL SNIL KIQR - H - E NIVQLVNV - - MKA ENN- - - - KD I Y L A F EYMET DLHAVI RANI - - L E D I Q I RY - H - E NIVKL L DV - - - L V P E D P - - - S NF D D Y V V F D F MQT DMHK I I S S KQ - D L S P D HMQY - H - DNI I RL FNV - - - L KAEND - . - - KDI Y L VFEF LDS DLHQVI KS N I - - LEDI HKRY - H - - NP F IVGI LD V - I RAAND - - - - I DL Y L VFEL I EA DL TA I I RKNL - - LQRDHKRF - H - P NI I KL LHV - H - - RAFND - - - - RDI Y L VF EYMET DLHVVI RAN - - LEE I HKQF - H - E NL L NVVNI - - - L P P L KR - E Y H S F E DVYVVT P L MDV DMNVVL R S RQ - V L E E S HMQY - H - N NL I RL HHF - - - MRPQSK - - E T F E D I Y L VMDL YDT DL NR I I R S RQ - K L T DE HLQY E C GCR NV L RL I RV - - - L P P RDP - - IME F RDL Y L VTDL YD I DL F S I I RQNK - C E S I D L L RR

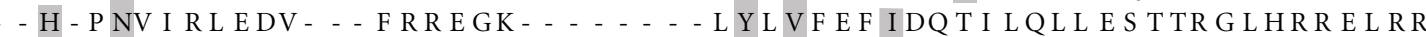
G - H - P NIVRLMEVGRP PAP TGAS S SAAF DDI Y L VTDLMDTDLGALL RS SQ- E I AMDQL R F

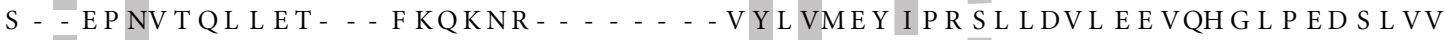

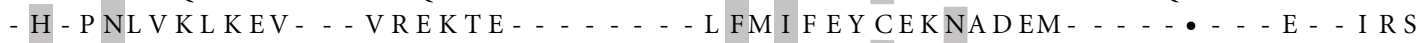
- H - P NI L GL RD I F - - VHF E E P - - - AMHK L Y L VTE L MRT DL AQV I HDQR I V I S PQH I QY D - - DNI I GLRNI - - L T PKDP - - ENF DHFY I VMD I ME T DL KQVL R S GQ - E L T E A H I QF - - H - P NI L S L KDL - - - F K S P DP - - VDTY S E L YVVTDL ME S DMDA I L R S PRI R L A AGHGQY P - H - P NIVDLVEV - - L F DR S T - . - - GRL A L VL E L MDM S L Y E L I KGRKQY L GE E KVRS G - H - P NVVKLREV - - I RENNE - - . - - L F F VF EYMDGDL LGVI KKA - - I PYP LVKN E A H - P HV I GY F S I _ . . . . - K T D E F N - VH I VMP L MKG DL F Y F I R L L . . . . . . . G - H - DNI I KLMDV - - - I KAKND - - - - - NDI Y L I F DF METDLHEVI KADL - - LE E I HKKY S - - D DI I RLHDL - - I I PED - - L L KF DELY I VLE I ADS DLKKLF KT P I - F L TEQHVKT - H - ENI I NLVEI - - - L DP L T P - - - D F E D I Y L VS DL MDT DLHRVI Y S RQ - P L T P E HHQY G - H - ENIVRLKNV - - L KADND - - - - KD I YLVF DYMET DL HAV I RAD I - L E E I HKQY - H - D HVVKVLDI - - - V I P KD - - - VEKF DE L YVVLE I ADS DF KKLF RT PV - Y L T E L H I KT ..++++++++ . ....++++++++++++++ . .

V
$+. .+.++$ VIa

FIgURE 1: Continued. 


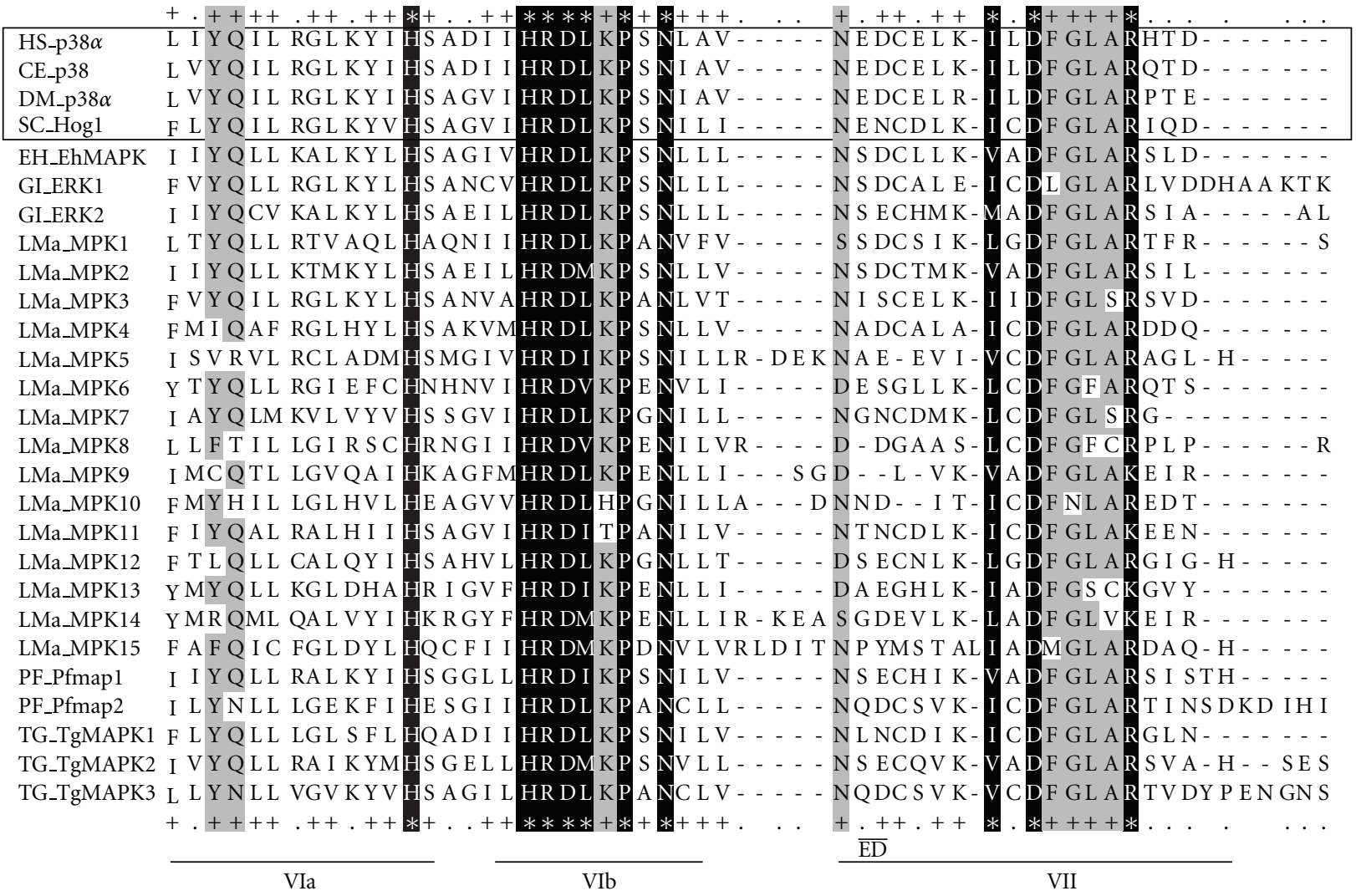

190 200 210 220 230

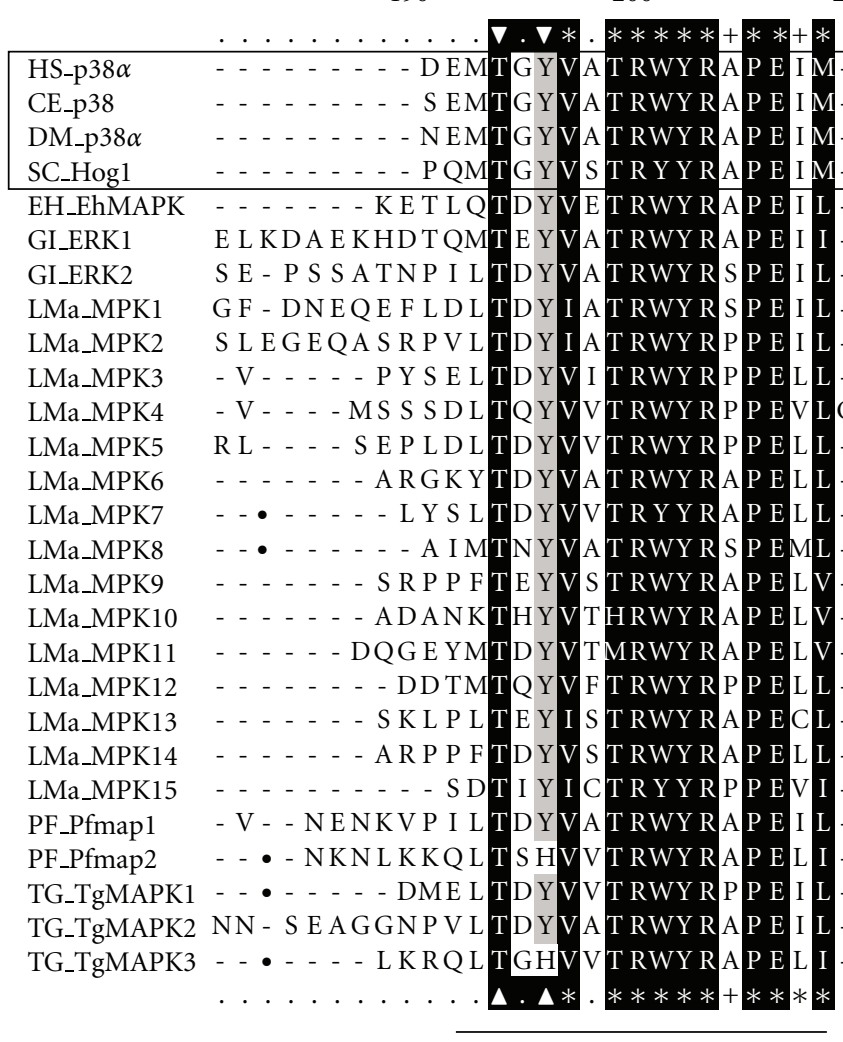

VIII

\section{$*+. . .+$}

+ . . $+* * *+.++*+$.**
L NWMHYNQT VD I WS V GC I MAE L L T GR T L F P G T L NWMHY T Q T VDVWS V GC I L A E L I T GK T L F P G S - L NWMHY DQT VD I WS V GC IMAE L I T R R T L F P G T L TWQK Y DV E VD I WS A GC I F A EM I E GK P L F P GK L - LGS QRYS F A I D LWS VGCI L GE I I NGKPLF PGS I - LGWPQYGK PVD I F S VGCI F AEL I ARK P L F P GR - L GCTRYT KGVDMWA I GC I L GEML GG S PMF P G S - VK S R A Y S T AMDMWA VGCV I GEML L GH P L F E GR - L G S T R Y T K GVDMWS VGC I L A E LML GK P I F P GR L ENTNYS T A VD I WS VGC I F A EMYNR K PVF P GR GMG S NQY T S A VDVWS L G L I F A E LMVG R A L L P G T - LMC P - Y S Y P I D I WA VGCVMAE Y AMQR P L F A GR - VGDVA Y GK P VDVWA L GCMF AEL S DGQP L F P GE - I - MGR YNHA I DMWS A GCI L A EMV L R R P L F T GA - L GMS S Y T Y A VDMWA VGA IMAEA I DGE P L L P GK - LHS THYN S P VD I WA CAVI F AEL Y L C R P LF P G T - MQ F K G F T K L VDMWS A G CVMAEMF NR K A L F R G S - MEDKDY S VQ I DVWG I GC I L GEL L GS R P L F QGK - L V CKHCNY S ADMWA V GCL A AEMF T GK P LF P GK - L TDGYYNY KMDLWS A GCVF FE I I A L F P L F P G S - LQDR F Y GA A VDVWA A GCIMVEL I TMR P LF P GT - T S V S GGS P R ID IWS L GCI F Y EMCT GQTL F TMR - L G S THY T E DVDMWS L GCIMGE L L CGK P LF T GN - LLQENYTNS IDIWS TGCIFAELL - - PLFPGS - I S P FCYS K P VDLWS VGCI F AE L L GRRAL FAGK - L G S T S Y T KGVDMWS L GCI L GEL L S GR P I F P GT - L LQENYTEA I DVWS I GCIFAELL-• P LFPGS $+. .+. .++* * *+.++*+. *+. .++*+++$.

\footnotetext{
Figure 1: Continued.
} 


\begin{tabular}{|c|c|c|c|}
\hline & $\begin{array}{l}250 \\
.+.\end{array}$ & $\begin{array}{l}260 \\
\ldots .\end{array}$ & $\begin{array}{l}270 \\
. .+++\end{array}$ \\
\hline HS_p38 $\alpha$ & DH I D - Q L K L I L R L V & $-\mathrm{T}_{2}-\cdots$ & GA - - E L LKK I S S E S A RN - - - - Y I Q S L - - TQMPK \\
\hline & H I D - Q L T R I MS V T & $\ldots \mathrm{T} \ldots \ldots$ & DE - - EF LKKI S SEEARN- - - - Y I RNL - PK - MTR \\
\hline DM_p38 & - HQ L N L I MEML & $-\mathrm{T}_{-} \ldots-$ & PA - - E F LKKI S S ESARS - - - - Y I Q S L - - P PMKG \\
\hline SC_H & V - HQF S I I T D L L & $\ldots S \ldots-\cdots$ & PK - - DV I NT I C S ENTLK - - - - FVTS L - PH - RDP \\
\hline EH_El & LN-QLDK I I E A T & $\cdots-\cdots Q$ & SA - - E D L E V I D S P L SMN- - - - L L S S L - PQ - RE T \\
\hline GI_E & I I - HQLHL I L E V L & $-\mathrm{T}_{-1}-\mathrm{C}_{1}$ & EK - - ELLDR I A S DSAKS - - - Y Y L A L - - KP S AP \\
\hline GI_E & TMN - QL D K I M - - - & - - - G - HWE R & TP - - ED I EATESPFA SM - - - MLDS L - - QP K T G \\
\hline $\mathrm{LMa}$ & T L D - Q L R L I V E A I & $\cdots-\cdots-V_{1}$ & S D - ADVRS LHS P E LET - - - - L INS L - - - P T P \\
\hline $\mathrm{Ma}$ & S T T N - Q L E L I C & $\ldots-\cdots M$ & PSA - - ADVAATNSQFAHA - - - - MLRDI - - HCAHR \\
\hline K3 & NTMD - QL RM I A QH I & 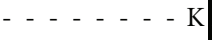 & PPA - - - S I VEHREALE - - - KLNEL - - P DGS \\
\hline & Y I - GQLVMI VN & $-S_{-} \ldots-\cdots$ & PS I - DDME F L S SE - AKA - - - F I L SQ - PH - R PA \\
\hline 5 & DY I - HQLQF V L - - - & - - S I - - - - & I T GVDF I ER S S S S S GLA - - - - NMNE I A KKYKGT \\
\hline $\mathrm{CMa}$ & S D L D - Q L C L I MQ & & VP - - - - QRLVF I FMHN - - P L YNG I S \\
\hline $\mathrm{LMa}$ & NYL - SQLA L I L & - L R G - VPQT & 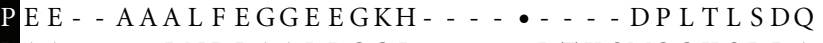 \\
\hline LMa & TE L - EQL S L I Q & $-\cdots-\cdots$ & PAA - - - L N P L A A P PQQL - - - - R T K SM \\
\hline LMa & S E S D - QL F K I C S V L & $--S_{-}-\cdots$ & A P - - NEWDE - GYQLAR R - - - - - MNMR F P T - VA P \\
\hline LMa & T F YN - QLNK I V E & $\cdots \mathrm{T} \ldots-\cdots$ & PKI - - EDVVMF S S P SARD - - - Y Y R R S - L S NVPA \\
\hline & D R VN - QLDK I VDV I & $-\mathrm{T}_{-1}-\mathrm{C}_{1}$ & PS E - - ED I NSVGS S AAQK - - - - Y L KKK - - SHRPQ \\
\hline & Y I N - Q I N L I V E L L & 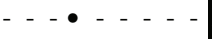 & PS KG - KKLEEYAP E LRRR F DETTF Y S F D T E L E E A \\
\hline Ma_Ml & NE L D - QVHR I HNV L & $\ldots$ T P - . - & PTE I LER L KK F G THMDYD - . . . . - . F P K - KQG \\
\hline LMa_M & NE VD - QL F K I MS V L & $-S_{-} \ldots-\cdots$ & PTE - - E EWA GG L R L A KK - - - - - I R Y T F P K - VAG \\
\hline LMa & E V V L N & $-\mathrm{T}_{-1}-\mathrm{C}_{1}$ & PAA - - ED I ERYMP S GNAK - - - LYLQRS - - A ARP \\
\hline PF_P & S TMN - Q I & $-\cdots-\cdots$ & PNK - - KD I ED I R S P FAEK- - - - I I S S F - - VDLKK \\
\hline PF_Pfmap2 & $-\bullet$ D - QLN I I FNVI & $-\mathrm{T}_{-}-\cdots$ & PP - - E E D L KC I T KQE V I KY I K L F P T R DG I D L S K KY \\
\hline $\mathrm{TG}_{-}$ & DHF D - QL R R I V R V L & $-\cdots \cdot-\cdots$ & PS KGT SNTKRKRSEAARR - - - - F I ES L - PN - S DP \\
\hline & S TMN - QL E R I MT L T & $-\cdots-\cdots$ & PS P - E EVDAVKS PFAAT - - - MMES L - - P LGKV \\
\hline TG_TgMAPK3 & $-\bullet$ D - QLNVIF & & PSE- - ED I EA L EKEDAKRY I R I F P KREGTDLAER F \\
\hline & & & . \\
\hline
\end{tabular}
A K E AMEHEY L A A Y HD $\underset{\sim}{\widetilde{N}} \widehat{T} \underset{\sim}{D} E$ A E E A L S HP Y L E KY A E P S V E A A D A L A H P Y S A P Y H $\mathscr{\sim}_{\mathcal{N}}$ P T DE A EKA LES T FVAD FHDPNDE VNEC L S H P Y F E G I HD̃ E S D E A EQA L EHP Y L S K F HD P A T E A V E A L QHP Y V A P F L Q P GE L A A E A L EHP YVAA F HR P DE E A A DML AHP Y L AH L HDE $\widetilde{\text { CDE }}$ A KQVMEHP Y F S KYR D A $\widetilde{A E}$ AQE A L KHP F F S S VG G P D $\widetilde{\sim} K$ C T E LME L P Y F T R DGF R D R Y A L E A L R D P F FWP L Y S $\widetilde{R} D E$ V E E A L GHP Y F D S VRGR F $\underset{\sim}{\sim} A$ A T QC LQHPY F T G S GG S S Ã L TEQA L RHP Y F E S F D P L D L V L QAMRHP F L EQ LH $\widetilde{\sim} D A \widetilde{D} D$ AKE S I AHAWL SDVR R PQE T AKE A L RHA Y F S K L R $\underset{\sim}{\mathrm{ADKK} K}$ A EQC L QHP F FNVG I $\stackrel{\sim}{D}$ E C $\mathrm{NA}$ AQQL CQHQL F RNYNV F Y GS A ENA L KHKYVEE FHS I I DE I DKA L S H P Y L KDVR K EN $\tilde{L} E$ VQEA L RHEY F E GLHS VEDE AEKGLEHPYVRQFHS P $\widetilde{E D E}$ I NEC L AHP F F KEVR I A $\widetilde{E V E}$ $.+.+.++++. .+{ }^{2} .{ }^{\aleph}++$ 


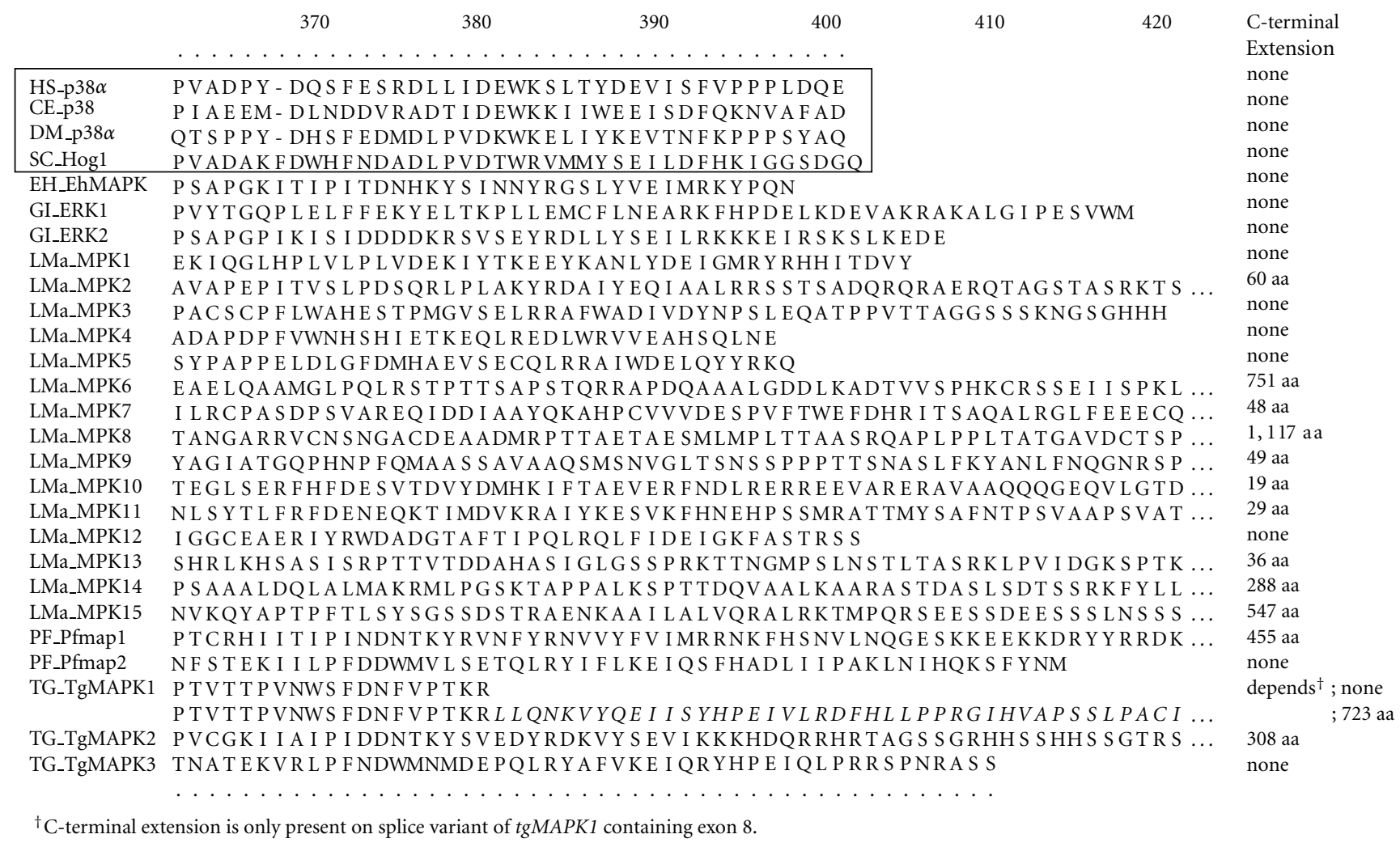

FIGURE 1: ClustalW alignment of representative MAPKs of diverse evolutionary origin, with each of the 11 subdomains indicated (Roman numerals). Conserved acidic residues within the ED site (subdomain VII) and common docking (CD) domain, which immediately follows subdomain XI, have been underlined. The first four sequences represent p38 MAPKs of metazoan and yeast origin and are boxed as reference sequences to which other protozoan MAPKs can be compared. Invariant MAPK residues (within allowed substitution groups) are highlighted in black and denoted by an asterisk. Highly conserved residues ( $>80 \%$ conservation) are highlighted in grey and denoted by a plus sign. In the absence of grey shading, plus signs indicate residues conserved in the majority of aligned sequences. Allowed substitution groups include acidic/amide (DE, DN, EQ), aliphatic (LIVM), aromatic (FYW), basic (KR), and hydroxyl/polar residues (STG). The positions of insertion sequences removed prior to ClustalW alignment are indicated by filled circles. White triangles denote the position of the TX[XY] phosphorylation lip. Two letter abbreviations precede the name of each MAPK sequence, indicating the genus and species of origin for each MAPK. CE: Caenorhabditis elegans; DM: Drosophila melanogaster; EH: Entamoeba histolytica; GI: Giardia intestinalis; HS: Homo sapiens; LMa: Leishmania major; PF: Plasmodium falciparum; SC: Saccharomyces cerevisiae; TG: Toxoplasma gondii. Accession numbers of all aligned sequences are listed in Tables 1 and 2.

in Plasmodium. Moreover, no P. falciparum MKK genes have been identified, suggesting that $P$. falciparum MAPK signaling does not utilize typical MAPK cascades [46]. $P$. falciparum Pfmap-2 is instead activated by Pfnek-1, a neverin-mitosis/ㅅspergillus- (NIMA-) related kinase [47]. Since homology amongst MKKs and MKKKs is much lower than that for members of the MAPK superfamily, it is conceivable that genes encoding these proteins exist but have simply not been annotated as such in the P. falciparum genome.

Pfmap-1 is neither required for schizogony nor gametocytogenesis in human erythrocytes cultured in vitro, nor for gametogenesis and/or sporogony in the mosquito vector [48]. However, Pfmap-2 protein levels are elevated in pfmap-1 knockout parasites, suggesting that Pfmap-1 fulfills an important function necessitating compensatory adaptation in parasites lacking this enzyme. Pfmap-2 is essential for the completion of the P. falciparum asexual cycle
[48]. Functional characterizations of MAPKs from $P$. vivax, P. ovale, and P. malaria, the other Plasmodium species causing malaria, have yet to be reported to our knowledge.

2.2. Genus Toxoplasma. T. gondii, the sole member of the genus Toxoplasma, can cause significant morbidity or mortality in hosts with compromised cellular immunity. Like $P$. falciparum, T. gondii appears to be another protozoan parasite that lacks typical MAPK activation cascades. Preliminary examination of the T. gondii genome suggests that it encodes four MAPKs. However, the TGME49_021550 locus (situated on chromosome II) lacks coding sequences corresponding to several essential MAPK motifs (including an incomplete MAPK signature sequence), thereby disqualifying it as a functional MAPK gene. Of the remaining three MAPK genes (Table 1, Figure 1), we have cloned and sequenced both the genes encoding $\operatorname{tg} M A P K 1$, situated on chromosome XI [50], and tgMAPK2 (chromosome VIII; 
TABLE 1: Non-Trypanosomatid mitogen-activated protein kinases discussed in this review.

\begin{tabular}{|c|c|c|c|c|c|c|}
\hline Organism & MAPK & Accession no. & $\begin{array}{l}\text { Phosphorylation } \\
\text { lip }\end{array}$ & Classification & Function & References \\
\hline Caenorhabditis elegans & p38 & AAB00664 & TGY & Typical & Stress-response & [36] \\
\hline Drosophila melanogaster & p $38 \alpha$ & AF035547 & TGY & Typical & Stress-response & {$[35]$} \\
\hline Entamoeba histolytica & EhMAPK & AY460178 & TDY & Typical & $?$ & \\
\hline \multirow{2}{*}{ Giardia intestinalis } & ERK1 & AY149274 & TEY & Typical & Encystation & {$[49]$} \\
\hline & ERK2 & AY149275 & TDY & Typical & Encystation & {$[49]$} \\
\hline Homo sapiens & p38 $\alpha$ & Q16539 & TGY & Typical & Stress-response & {$[21,34]$} \\
\hline \multirow{2}{*}{ Plasmodium falciparum } & Pfmap-1 & Q94656 & TDY & Typical & $?$ & \\
\hline & Pfmap-2 & Q25917 & TSH & Atypical & $\begin{array}{l}\text { Essential for } \\
\text { differentiation }\end{array}$ & {$[48]$} \\
\hline Saccharomyces cerevisiae & Hog 1 & AAA34680 & TGY & Typical & Stress-response & {$[6]$} \\
\hline \multirow[t]{3}{*}{ Toxoplasma gondii } & $\begin{array}{l}\text { TgMAPK1 } \\
\text { (BARKY) }\end{array}$ & AY684849 & TDY & Typical & $\begin{array}{c}\text { Proliferation }^{\dagger} \text {, } \\
\text { differentiation }^{\dagger} \text {, } \\
\text { virulence }^{\dagger}\end{array}$ & \\
\hline & TgMAPK2 & DQ115400 & TDY & Typical & $?$ & \\
\hline & TgMAPK3 & XP_0022369585 & TGH & Atypical & ? & \\
\hline
\end{tabular}

${ }^{\dagger}$ Brumlik et al., submitted.

[18]). We have also sequenced the third MAPK gene, tgMAPK3 (chromosome Ib).

TgMAPK1 is a critical virulence determinant during acute T. gondii infection (Brumlik et al., submitted). By expressing it in Hog1-deficient yeast lacking its own stress-response MAPK, we restored yeast ability to grow under osmotic stress [50], providing evidence for this MAPK's role as a stress-response MAPK. Since TgMAPK1 expression affects tachyzoite/bradyzoite stage differentiation (manuscript in preparation), we renamed it "BARKY" (bradyzoite antigen regulator, kinase $\mathrm{Y}$ ).

BARKY is a typical MAPK based on conventional criteria [50] although it possesses three insertion sequences. Using mass spectroscopy, we confirmed the presence of a 34 amino acid insert situated between the GXGXXGXV motif (subdomain I) and the invariant lysine residue within the VAXK motif of subdomain II, a region responsible for anchoring the nontransferable $\alpha$ - and $\beta$-phosphates of ATP during catalysis. $B A R K Y$ is also predicted to encode a 93 amino acid insert situated between the DFGLAR motif that interacts with the $\mathrm{Mg}^{++}$bound to ATP and the phosphorylation lip, which links the proline-directed peptide binding pocket in an extended conformation following phosphorylation of its activation loop (subdomains VII and VIII, resp.). Finally, using mass spectroscopy, we identified a 20 amino acid insert between subdomains IX and X.

Phylogenetic analysis demonstrates that T. gondii BARKY most closely resembles Cryptosporidium hominis MAPK (with $52 \%$ amino acid sequence identity across all 11 of the MAPK subdomains), with a corresponding homologue in C. parvum. No other closely related MAPK homologues were identified either within or outside the phylum Apicomplexa at the time of publication [18].

Alternative splicing within exons 3-4 and exons 7-8 of the BARKY gene results in multiple BARKY isoforms, producing protein variants that could differentially respond to upstream signals or have altered substrate specificity. In support, we have detected 50,58, and $\sim 130 \mathrm{kDa}$ proteins in T. gondii tachyzoite cell-free extracts by Western blotting. We have also employed mass spectroscopy to detect peptide fragments that confirm the existence of the full length $(130 \mathrm{kDa})$ BARKY protein in tachyzoites grown in vitro. We cannot exclude the possibility that the smaller forms of the protein result from proteolytic degradation, but reverse transcriptase-polymerase chain reaction has demonstrated the presence of BARKY transcripts with a stop codonsituated 84 nucleotides into exon 7 , as well as an alternative $B A R K Y$ splice variant encoding exon 8 that adds a 766 amino acid extension to the carboxy-terminus (Brumlik et al., unpublished observations). These features are reminiscent of extensions identified in many Leishmania MAPKs [51].

There are no reported functional data for $T$. gondii TgMAPK2 but it is expressed in T. gondii tachyzoites at the expected molecular weight of $73 \mathrm{kDa}$ (Brumlik et al., unpublished observations). Phylogenetic analysis places this MAPK in a group of closely related Apicomplexan MAPKs which includes Cryptosporidium hominis MAPK1, P. falciparum Pfmap-1, and Theileria annulata MAPK (all sharing roughly $70 \%$ amino acid sequence identity across all 11 of the MAPK subdomains). TgMAPK2 shares significant amino acid sequence identity with MAPKs from non-Apicomplexan protozoans including L. mexicana LmxMPK2 (62\%) and Trypanosoma brucei TbMAPK2 (62\%), each possessing a typical TDY phosphorylation lip. The deduced amino acid sequence of TgMAPK2 shares $55 \%$ identity with human ERK8 across all 11 MAPK subdomains, demonstrating the remarkable evolutionary conservation of this MAPK subfamily member. In addition, T. gondii TgMAPK2 possesses multiple copies of a VSSSHHG repeat in its carboxyterminal extension, the exact number of repeats being straindependent [18]. While the role of this repeat remains unknown, it is striking that $P$. falciparum Pfmap-1 possesses 
an analogous series of imperfect KKYVD[GSE][GSL]N repeats in its carboxy-terminal extension [43]. Short amino acid repeats often facilitate oligomerization or serve as contact points for protein-protein interactions. Interestingly, TgMAPK2 is also predicted to possess a nuclear localization signal within its carboxy-terminal extension.

T. gondii TgMAPK3 is predicted to be an atypical $63 \mathrm{kDa}$ MAPK with a TGH phosphorylation lip. It shares significant amino acid sequence identity with several Apicomplexan MAPKs such as Cryptosporidium hominis MAPK2 (67\%), P. falciparum Pfmap-2 (58\%), and Theileria annulata MAPK2 (50\%), with low amino acid sequence identity to non-Apicomplexan MAPKs [18].

\section{Phylum Sarcomastigophora}

3.1. Trypanosomatid MAPKs. Trypanosomatids (members of the family Trypanosomatidae) are a diverse group of protozoan parasites of which two genera are human pathogens: Trypanosoma and Leishmania.

\subsubsection{Genus Leishmania. Several different Leishmania} species cause human disease of varying clinical presentation and severity, of which $L$. major generally causes the most serious illnesses. Genome sequencing has identified 15 putative complete MAPK genes in L. major (Table 2), the alignments of which are shown in Figure 1. Two partial $L$. major MAPK genes have also been identified (LmjF03.0210 and LmjF13.07800) [52] but have been excluded from further consideration because they lack the coding region for the complete MAPK signature sequence. All 15 L. major MAPK homologues have also been identified in L. mexicana (Table 2), L. infantum, and L. brasiliensis [52].

Each of the 15 unique Leishmania MAPKs (Figure 1) is a typical MAPK by the classical definition (i.e., the activation loop is comprised of a TXY motif). The majority of these MAPKs possess carboxy-terminal extensions (Figure 1), some of them over 1000 amino acids long (as for LmaMPK8). This region may be analogous to the corresponding region of human ERK5 or ERK8, each of which possesses a C-terminal transactivation domain and nuclear localization signal [22, 23]. LmaMPK6, 7, and 8 are predicted to contain nuclear localization signals within their carboxy-terminal extensions, making them even more closely resemble human ERK5 and ERK8, as well as T. gondii TgMAPK2.

Deletion analysis of the genes encoding L. mexicana LmxMPK1 and LmxMPK2 demonstrates that both are essential for amastigote (bloodstream stage) survival [52, 53]. L. mexicana LmxMPK4 is essential to both promastigote (sandfly stage) and amastigote forms [58] and is phosphorylated on $\mathrm{T}^{190}$ and $\mathrm{Y}^{192}$ of its phosphorylation lip by the MKK LmxMKK5 [64]. Overexpression of L. major LmaMPK4, 7, or 10 (homologues of LmxMPK4, 7, and 10, resp.) causes stage-specific induction of phosphotransferase activity. Moreover, LmaMPK7 activation specifically regulates parasite growth [62]. In each case, kinase activity was low or absent in cell-free extracts from promastigotes but significantly increased after exposure to $\mathrm{pH} 5.5$ and $34^{\circ} \mathrm{C}$., which simulates the stress encountered by the parasite in the acidified phagolysosome upon invasion of macrophages [59]. L. mexicana LmxPK4 is an MKK that controls parasite differentiation [65] and thus represents a potential upstream activator of at least one of the MAPKs affecting stage differentiation.

Several L. mexicana MAPKs regulate flagellar length, many of which possess carboxy-terminal extensions [66]. Deletion mutants for LmxMPK3 had shortened flagella and overexpression of LmxMPK3 in the deletion background complemented this defect $[56,57]$. Deletion mutants for LmxMPK9, LmxMPK13, or LmxMPK14 generated promastigotes with elongated flagella, an effect that could be reversed by overexpressing these MAPKs in null mutants $[57,63]$. LmxMPK13 is the homologue of LF4 from the protozoan microalga Chlamydomonas reinhardtii, which also regulates flagellar length [67]. L. mexicana LmxMKK is the MAPKK responsible for regulating flagellar length [68] and activates LmxMPK3 [56] and perhaps affects other MAPKs regulating flagellar length.

Analysis of the L. mexicana genome has identified two additional putative MKK genes in addition to $L$. mexicana $\operatorname{lm} x P K 4, \operatorname{lm} x M K K$, and $\operatorname{lm} x M K K 5$ for which functions have yet to be determined. L. mexicana also putatively encodes 23 MKKKs and a single MKKKK [51], the functions of which remain unknown.

3.1.2. Genus Trypanosoma. Subspecies of T. brucei cause African sleeping sickness, whereas T. cruzi causes New World trypanosomiasis (Chagas disease). Genomic sequencing has identified 13 MAPK genes in T. brucei, each of which has at least one, but often two virtually identical copies of MAPK homologues in T. cruzi (with each copy having greater than $99 \%$ amino acid sequence identity to the other (Table 2)) [51, 52]. Homologous T. brucei or T. cruzi MAPK domains are $\sim 90 \%$ identical to each other and each has a single corresponding homologue in Leishmania spp. (sharing over $80 \%$ amino acid sequence identity across the 11 MAPK subdomains). LmxMPK7 and LmxMPK8 are the only two Leishmania MAPKs that lack homologues in either T. brucei or T. cruzi. Thus we exclusively used the L. major MAPK sequences (LmaMPK1-15) for ClustalW alignment, reducing redundant examples of highly homologous MAPKs in the analysis.

Although all Trypanosoma MAPKs possess a classical TXY motif, a feature also conserved in all Leishmania MAPK homologues (Table 2), the central amino acid in the TXY motif varies between MAPK homologues from different Trypanosomatid species (Table 2) and thus is not as evolutionarily constrained as in mammalian MAPKs.

T. brucei/cruzi MPK10 (accession nos. Q580Z7/ Q4D4Q4) and MPK11 (accession nos. Q389D8/Q4CZQ7) have not yet been officially named (see Table 2). Regardless, these MAPKs (and their Leishmania MAPK homologues) are exceptional in possessing a MAPK signature sequence that deviates with respect to the precise position of the threonine in the proline-directed $(\mathrm{P}+1)$ peptide binding pocket (see Figure 1, center of subdomain VIII, residues 197-207). This likely alters the precise spatial orientation 


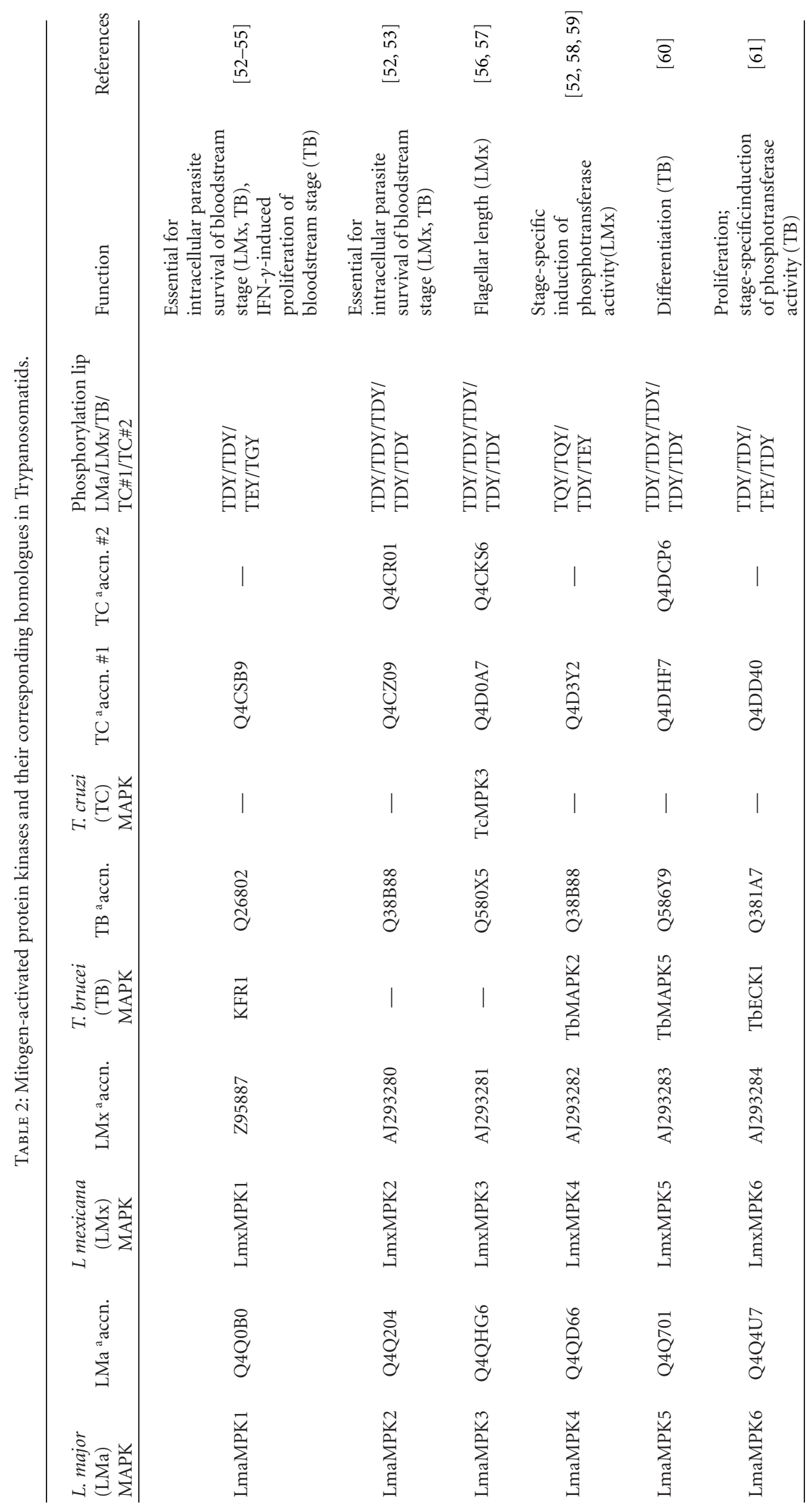




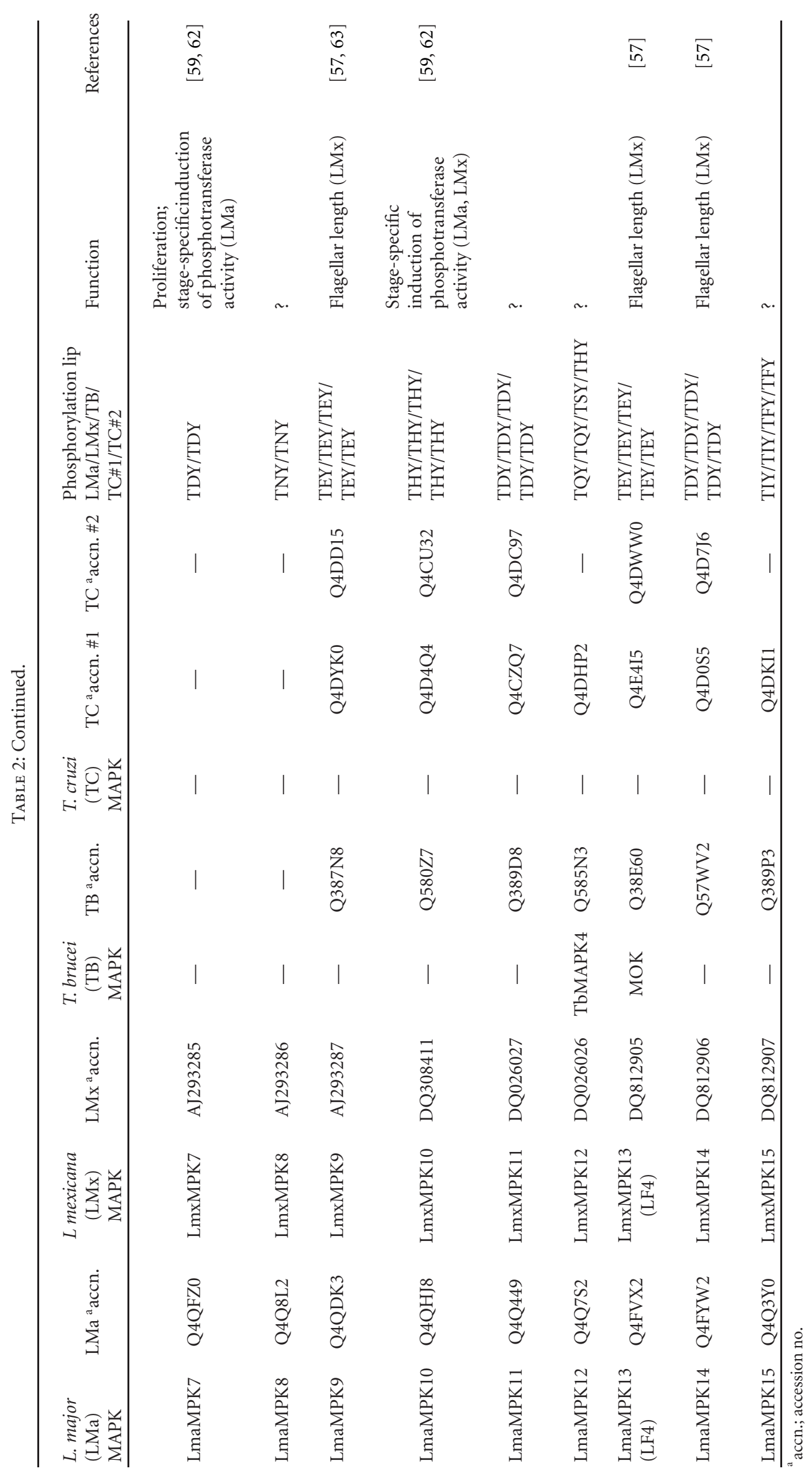


of the proline-directed peptide binding pocket relative to the phosphorylation lip, perhaps placing these MAPKs in a separate subfamily.

KFR1 (KSS1- and FUS3-related kinase 1), the T. brucei homologue of L. mexicana LmxMPK1, mediates interferon$\gamma$-induced amastigote proliferation and phosphorylates serine residues on host histone $\mathrm{H} 1$, myelin basic protein, and $\beta$-casein $[54,55]$. T. brucei TbECK1, which is the trypanosome homologue of $L$. mexicana LmxMPK6, possesses a carboxy-terminal extension that regulates kinase activity in all life cycle stages. Expression of a truncated TbECK1 protein lacking large parts of this extension caused T. brucei to grow slowly with abnormal morphology [61]. T. brucei procyclic forms lacking TbMAPK5, the homologue of L. mexicana LmxMPK5, likewise showed impaired differentiation into the bloodstream form [60]. TbMAPK2, the T. brucei homologue of LmxMPK4, regulates cell cycle progression from the procyclic (tsetse fly midgut) form to the bloodstream form [69]. TbMAPK5 controls T. brucei differentiation [60]. No functional studies of T. cruzi MAPKs have been published to date to our knowledge.

Phylogenetic analysis of T. brucei and T. cruzi suggests a single gene orthologous to the five putative MKK genes in L. major [51]. Only about one-third of the putative L. major MKKK genes have phylogenetic branching patterns consistent with the existence of orthologous genes in T. brucei and T. cruzi [51]. In most cases, L. major and T. brucei MKKK genes appear to be paralogues, having arisen from gene duplication events [51], suggesting significant evolutionary divergence in the circuitry of signaling cascades in Trypanosomatids. Three unique MKKKK genes have been identified in T. cruzi and two in T. brucei [51]. Functions have yet to be ascribed to any of these putative upstream MAPK activators.

3.2. Other Sarcomastigophora. Two MAPKs have been identified and characterized in the protozoan intestinal parasite Giardia lamblia, ERK1 and ERK2 (Table 1, Figure 1), each of which plays distinct roles in encystation [49]. In addition, one MAPK gene has been identified in the Trichomonas vaginalis genome [70]. However, functional studies have yet to be performed on MAPKs from either parasite.

3.3. Subphylum Sarcodina (the Amoebae). This subphylum of amoebas contains three human pathogenic genera: Entamoeba, Naegleria, and Acanthamoeba. The E. histolytica EhMAPK gene encodes a putative MAPK with significant homology to human ERK8 [71]. We are not aware of any further MAPK analyses in this genus or of any reports of MAPK genes or function in Naegleria or Acanthamoeba.

\section{Protozoan MAPKs as Therapeutic Targets}

MAPKs direct many functions critical to pathogen homeostasis and survival, including proliferation [62], differentiation $[52,53]$, regulation of cytoskeletal features such as the biosynthesis of flagella [56, 57, 63], and stress-responses [50]. Because protozoan MAPKs share many common structural features and are vastly more closely related to each other than to human MAPKs $[18,72]$, it should be possible to design drugs specifically or preferentially targeting protozoan MAPKs. For example, Leishmania mexicana LmxMPK1 and LmxMPK2 are essential MAPKs required for differentiation [52, 53], with corresponding homologues in other Leishmania species and in T. brucei and T. cruzi [51], but bearing scant resemblance to human MAPKs, making them excellent candidates for drug development [72]. Specifically targeting these MAPKs could have far reaching therapeutic potential since one drug could be used to treat a broad range of Trypanosomatid infections based on the high degree of homology between Trypanosomatid MAPKs [51].

P. falciparum Pfmap-2 is likewise an excellent druggable target as this MAPK is essential for the parasite to complete asexual replication in infected human erythrocytes [48] and it is highly dissimilar to human MAPKs. Although we have yet to determine which of the T. gondii MAPKs are essential to parasite survival, reducing BARKY expression dramatically impairs parasite virulence (Brumlik et al., submitted), making BARKY a useful target for MAPK inhibitor drugs.

Agents interfering with the function of MAPKs that affect stage differentiation, such as T. gondii BARKY, or affect parasite growth, such as L. major LmaMPK7 or T. brucei TbECK1, likely would be useful antiparasitic agents. T. brucei KFR1 is an interesting MAPK target, as it regulates effects of the host immune response (interferon- $\gamma$-induced amastigote proliferation) and could be considered in combination with an immune strategy. L. mexicana LmxMPK1 is homologous to KFR1 and could mediate similar effects, being a useful drug discovery target in this respect. Agents impairing the function of MAPKs controlling flagellar development or function, such as LmxMPK3, LmxMPK9, LmxMPK13, or LmxMPK14, could inhibit parasite dissemination and might be useful alone, or in combination with parasiticidal agents.

Our work with T. gondii BARKY demonstrates multiple MAPK splice variants that can occur naturally in parasites. A better understanding of the function of these splice variants could help develop agents specifically targeting variants relevant to disease pathogenesis. Likewise, our genomic analyses, and those of others, have demonstrated unusual repeat motifs in several protozoan parasite MAPKs (including in T. gondii and Plasmodium species) encoding large numbers of potential phosphorylation sites. An understanding of the functional significance of these motifs could help develop useful antiparasitic agents. Given the relatively unique nature of the phosphorylation site repeat motifs, these sites possibly could lead to highly parasite-specific drugs.

Protozoan MAPKs need not subserve critical functions to be useful drug discovery targets. For example, L. mexicana LmxMPK6 affects parasite morphology (which has indirect consequences on its growth rate following infection) and has homologues in related disease-causing Trypanosomatids. Drugs impairing LmxMPK6 function could be used in conjugation with existing anti-Leishmania therapies to boost their efficacy and could have broad-spectrum effects. 
Upstream components of the MAPK cascades such as the MKKs or MKKKs in pathogenic protozoan parasites are also potentially useful drug discovery targets. For example, the L. mexicana MKK, LmxPK4, controls parasite differentiation and thus is an excellent candidate. Because protozoan MKKs and MKKKs are even more distantly related to mammalian counterparts than MAPKs, a further potential advantage to this approach is that drugs inhibiting parasite MKK function could be less likely to have undesirable side-effects compared to drugs targeting specific MAPKs.

A potential disadvantage to targeting upstream MAPK regulators relates to our incomplete understanding of how they function. For example, MAPKs such as human p38 $\alpha$ are capable of MKK-independent activation and can undergo autophosphorylation in the presence of transforming growth factor- $\beta$-activated protein kinase 1 [73]. In this case, it would not be possible to block $\mathrm{p} 38 \alpha$ activation by targeting the conventional upstream MKKK and MKK components of the p38 MAPK cascade.

Many protozoan MAPKs possess vestiges of the common docking (CD) domain and ED site (Figure 1)—surfaceexposed acidic residues in human p $38 \alpha$ MAPK that facilitate binding to upstream and downstream MAPK partners [74, $75] . D^{313}, D^{315}$, and $D^{316}$ comprise the CD domain in human p38 $\alpha$ MAPK. This region acts in concert with the $\mathrm{ED}^{161}$ site to bind to short strings of $2-5$ basic amino acids situated on proteins with which p38 $\alpha$ interacts [74]. Protozoan MAPKs lacking a conserved CD domain (e.g., Leishmania major LMaMPK9 and 15) and/or ED site (e.g., Leishmania major LMaMPK3, 7, 9, 11, 15, and T. gondii TgMAPK1) are prime candidates for drug development since these domains have diverged considerably from their corresponding mammalian counterparts.

In addition, the highly variable carboxy-terminal extensions, which are present in over half the protozoan MAPKs shown in Figure 1, are excellent targets for drug development owing to their unique structures. Drugs targeted to these extensions would have a low probability of affecting mammalian MAPKs.

SB203580 is a pyridinylimidazole competitive ATP inhibitor affecting human p38 MAPK phosphotransferase activity through hydrogen bonding between its pyridine ring nitrogen and the MAPK backbone amide of $\underline{M}^{109}$ in the THLM ${ }^{109}$ motif (subdomain V; Figure 1) [39]. A second critical hydrogen bond occurs between a nitrogen atom on the imidazole ring and the invariant lysine in the $\mathrm{VAXK}^{53}$ motif (subdomain II). Finally, the fluorophenyl ring of SB203580 interacts with the hydrophobic environment created by $\mathrm{T}^{106}$ and $\mathrm{H}^{107}$ [39].

Because SB203580 is much smaller than ATP (as are all pyridinylimidazole p38 MAPK inhibitors), it does not fully occupy this region, leaving two large hydrophobic pockets on either side of the pyridine ring [39]. By designing novel pyridinylimidazoles or structurally related pharmacophores that properly fill the ATP binding pocket of pertinent protozoan MAPKs, it could be possible to develop novel antiparasitic agents that are more potent and specific than existing drugs. Such drugs will be less likely to have unintended consequences on host p38 MAPK, which is a potential drawback of several existing p38 MAPK inhibitors.

Recent molecular modeling studies using competitive ATP inhibitors against LCRK3 in L. donovani, a cyclindependent kinase that is a distant relative of the MAPK superfamily, indicate that such compounds could have significant inhibitory activity against $L$. donovani LCRK3 [76]. Our work has shown that the human p38 MAPK inhibitors RWJ67657, RWJ68198, and SB203580 reduced the replication of $L$. donovani promastigotes in axenic culture. Moreover, SB203580 effectively inhibited the replication of the bloodstream stage cultured ex vivo (Brumlik et al., unpublished observations).

X-ray crystallographic studies of human p38 $\alpha$ MAPK complexed with ATP have demonstrated that the THLM ${ }^{109}$ motif in the center of subdomain V (Figure 1) forms two critical hydrogen bonds with the adenosine moiety [77]. Based on our ClustalW alignment, many other amino acids can evidently serve this same purpose in other MAPKs (Figure 1; subdomain V), although the binding affinity of ATP (and competitive ATP inhibitor drugs) could be affected by such differences. Structural studies have further shown that the invariant GXGXXGXV ${ }^{38}$ motif in subdomain I coordinates the nontransferable $\alpha$ - and $\beta$-phosphates of ATP, while catalytic transfer of the $\gamma$-phosphate is mediated by hydrogen bonding between an essential lysine in the $\mathrm{VAXK}^{53}$ motif (subdomain II), the RE ${ }^{68}$ motif in subdomain III, and the underlined residues in the $\mathrm{HRD}^{168} \mathrm{XK}^{170} \mathrm{PXN}^{173}$ motif (subdomain VIb) [78]. Thus, to design novel competitive ATP inhibitors against protozoan MAPKs, one must not only account for the invariant residues comprising the ATP binding site in all MAPKs but also pay particular attention to the permissible structural changes in subdomain $\mathrm{V}$ of protozoan MAPKs that specifically affect the binding of competitive ATP inhibitors.

We have shown that SB203580 [50] and another pyridinylimidazole human p38 MAPK inhibitor, RWJ67657, significantly inhibit BARKY autophosphorylation (Brumlik et al., unpublished observations). These agents reduced $T$. gondii proliferation in vitro [41] and treated otherwise fatal T. gondii infection in mice [42]. We further assessed the efficacy of two human p38 MAPK inhibitors to treat parasitic infections and showed that RWJ67657 and the pyrrolobenzimidazole RWJ68198 effectively blocked the replication of $P$. falciparum cultured in human erythrocytes ex vivo. Drug treatment resulted in trophozoites that were markedly diminished in size (Brumlik et al., submitted).

We demonstrated that RWJ67657 protected mice from otherwise fatal infection with the protozoan Encephalitozoon cuniculi [42] although it encodes no known MAPKs. Inhibition of host p38 MAPK could improve the host immune response to E. cuniculi, as has been demonstrated for T. gondii [79], or RWJ67657 could have therapeutic off-target effects in either the host or parasite. Better understandings of the mechanism of action in this case will further help drug development.

A large number of p38 MAPK inhibitors have recently progressed into phase I and II clinical trials, thus providing 
basic inhibitor pharmacophores that can be modified to target critical protozoan MAPKs specifically while at the same time having less host toxicity (a problem with many agents in the pyridinylimidazole class).

\section{Conclusions}

MAPKs play essential roles in virtually all eukaryotes. Thus, inhibiting protozoan MAPK functions represents a scientifically sound approach to developing novel classes of antiprotozoan agents. As protozoan MAPKs are only distantly related to mammalian MAPKs and have distinct active sites, it is reasonable to expect that selective agents can be developed to target pathogen proteins with minimal collateral effects on human counterparts.

Although only a very modest body of work on the structure and function of protozoan MAPKs currently exists, the available evidence already suggests the general utility of inhibiting protozoan parasite MAPK function as a treatment strategy. Several specific MAPK candidates have also already emerged from such work. As interest in MAPKs increases, the rate of important discoveries and their preclinical and clinical translation will also increase.

Specific roles for MAPKs cannot be predicted based solely on sequence similarity to protein homologues. For example, P. falciparum MAPK Pfmap-2 is essential for the completion of asexual replication in human erythrocytes [48] and yet its closest homologue in P. berghei, Pbmap-2 (with 93\% amino acid sequence identity within its catalytic domains to Pfmap-2 [80]), is dispensable for both asexual replication and gametocyte formation in the mouse erythrocyte. Pbmap-2 instead plays a critical role in exflagellation in the mosquito midgut [81]. Thus, while the structure of the MAPKs themselves remains highly evolutionarily constrained even among closely related Plasmodium species, the circuitry of the various signal transduction pathways themselves has undergone significant divergent evolution. Therefore it is critical to establish specific roles of particular MAPKs prior to drug development.

Once the function of a MAPK from a pathogenic protozoan parasite has been established, one can exploit the phylogenetic differences between MAPKs of protozoan and metazoan origin to design specific MAPK inhibitors. Refining the structure of human MAPK inhibitor pharmacophores already in existence should speed development of new MAPK-inhibiting antiprotozoan drugs. We also expect to see additional new classes of drugs developed, which will be aided by additional structure/function studies. Targeting upstream MAPK regulators is an approach that also bears investigation, but which will likely lag owing to significant current knowledge gaps in understanding these regulators.

A considerable challenge is to persevere with such research given the relatively scant resources available for such work, in spite of the fact that over one-half billion people in many of the poorest parts of the world are infected by pathogenic protozoan parasites [82].

\section{References}

[1] S. Bagrodia and R. A. Cerione, "PAK to the future," Trends in Cell Biology, vol. 9, no. 9, pp. 350-355, 1999.

[2] G. Pearson, F. Robinson, T. B. Gibson et al., "Mitogenactivated protein (MAP) kinase pathways: regulation and physiological functions," Endocrine Reviews, vol. 22, no. 2, pp. 153-183, 2001.

[3] L. Chang and M. Karin, "Mammalian MAP kinase signalling cascades," Nature, vol. 410, no. 6824, pp. 37-40, 2001.

[4] D. Miranda-Saavedra, M. J.R. Stark, J. C. Packer, C. P. Vivares, C. Doerig, and G. J. Barton, "The complement of protein kinases of the microsporidium Encephalitozoon cuniculi in relation to those of Saccharomyces cerevisiae and Schizosaccharomyces pombe," BMC Genomics, vol. 8, article 309, 2007.

[5] D. Kültz and M. Burg, "Evolution of osmotic stress signaling via MAP kinase cascades," Journal of Experimental Biology, vol. 201, no. 22, pp. 3015-3021, 1998.

[6] D. Kültz, "Phylogenetic and functional classification of mitogen- and stress- activated protein kinases," Journal of Molecular Evolution, vol. 46, no. 5, pp. 571-588, 1998.

[7] S. K. Hanks, "Genomic analysis of the eukaryotic protein kinase superfamily: a perspective," Genome Biology, vol. 4, no. 5, article 111, 2003.

[8] P. Coulombe and S. Meloche, "Atypical mitogen-activated protein kinases: structure, regulation and functions," Biochimica et Biophysica Acta, vol. 1773, no. 8, pp. 1376-1387, 2007.

[9] B. J. Canagarajah, A. Khokhlatchev, M. H. Cobb, and E. J. Goldsmith, "Activation mechanism of the MAP kinase ERK2 by dual phosphorylation," Cell, vol. 90, no. 5, pp. 859-869, 1997.

[10] S. K. Hanks and A. M. Quinn, "Protein kinase catalytic domain sequence database: identification of conserved features of primary structure and classification of family members," Methods in Enzymology, vol. 200, pp. 38-62, 1991.

[11] C. Widmann, S. Gibson, M. B. Jarpe, and G. L. Johnson, "Mitogen-activated protein kinase: conservation of a threekinase module from yeast to human," Physiological Reviews, vol. 79, no. 1, pp. 143-180, 1999.

[12] I. Dan, N. M. Watanabe, and A. Kusumi, "The Ste20 group kinases as regulators of MAP kinase cascades," Trends in Cell Biology, vol. 11, no. 5, pp. 220-230, 2001.

[13] J. Avruch, "MAP kinase pathways: the first twenty years," Biochimica et Biophysica Acta, vol. 1773, no. 8, pp. 1150-1160, 2007.

[14] R. Seger and E. G. Krebs, "The MAPK signaling cascade," FASEB Journal, vol. 9, no. 9, pp. 726-735, 1995.

[15] D. Edbauer, D. Cheng, M. N. Batterton et al., "Identification and characterization of neuronal mitogen-activated protein kinase substrates using a specific phosphomotif antibody," Molecular \& Cellular Proteomics, vol. 8, no. 4, pp. 681-695, 2009.

[16] S. H. Yang, A. D. Sharrocks, and A. J. Whitmarsh, "Transcriptional regulation by the MAP kinase signaling cascades," Gene, vol. 320, pp. 3-21, 2003.

[17] Y. Miyata and E. Nishida, "Distantly related cousins of MAP kinase: biochemical properties and possible physiological functions," Biochemical and Biophysical Research Communications, vol. 266, no. 2, pp. 291-295, 1999.

[18] M. R. Lacey, M. J. Brumlik, R. E. Yenni, M. E. Burow, and T. J. Curiel, "Toxoplasma gondii expresses two mitogen-activated protein kinase genes that represent distinct protozoan subfamilies," Journal of Molecular Evolution, vol. 64, no. 1, pp. 4-14, 2007. 
[19] S. Yoon and R. Seger, "The extracellular signal-regulated kinase: multiple substrates regulate diverse cellular functions," Growth Factors, vol. 24, no. 1, pp. 21-44, 2006.

[20] J. M. Kyriakis, P. Banerjee, E. Nikolakaki et al., "The stressactivated protein kinase subfamily of c-jun kinases," Nature, vol. 369, no. 6476, pp. 156-160, 1994.

[21] A. J. Whitmarsh, "A central role for p38 MAPK in the early transcriptional response to stress," BMC Biology, vol. 8, article $47,2010$.

[22] G. Zhou, . Zhao Qin Bao, and J. E. Dixon, "Components of a new human protein kinase signal transduction pathway," Journal of Biological Chemistry, vol. 270, no. 21, pp. 1266512669, 1995.

[23] J. D. Lee, R. J. Ulevitch, and J. Han, "Primary structure of BMK1: a new mammalian MAP kinase," Biochemical and Biophysical Research Communications, vol. 213, no. 2, pp. 715724, 1995.

[24] H. Enslen, D. M. Brancho, and R. J. Davis, "Molecular determinants that mediate selective activation of p38 MAP kinase isoforms," The EMBO Journal, vol. 19, no. 6, pp. 13011311, 2000.

[25] M. Cheng, T. G. Boulton, and M. H. Cobb, "ERK3 is a constitutively nuclear protein kinase," Journal of Biological Chemistry, vol. 271, no. 15, pp. 8951-8958, 1996.

[26] M. Yamada, J. Ohnishi, B. Ohkawara et al., "NARF, an Nemolike kinase (NLK)-associated ring finger protein regulates the ubiquitylation and degradation of $\mathrm{T}$ cell factor/lymphoid enhancer factor (TCF/LEF)," Journal of Biological Chemistry, vol. 281, no. 30, pp. 20749-20760, 2006.

[27] B. O. Zhou and Z. Y. Zhang, "The activity of the extracellular signal-regulated kinase 2 is regulated by differential phosphorylation in the activation loop," Journal of Biological Chemistry, vol. 277, no. 16, pp. 13889-13899, 2002.

[28] B. W. Zanke, E. A. Rubie, E. Winnett et al., "Mammalian mitogen-activated protein kinase pathways are regulated through formation of specific kinase-activator complexes," Journal of Biological Chemistry, vol. 271, no. 47, pp. 2987629881, 1996.

[29] P. S. Swain and E. D. Siggia, "The role of proofreading in signal transduction specificity," Biophysical Journal, vol. 82, no. 6, pp. 2928-2933, 2002.

[30] M. K. Abe, M. P. Saelzler, I. I. I. Rafael Espinosa et al., "ERK8, a new member of the mitogen-activated protein kinase family," Journal of Biological Chemistry, vol. 277, no. 19, pp. 1673316743, 2002.

[31] M. K. Abe, K. T. Kahle, M. P. Saelzler, K. Orth, J. E. Dixon, and M. R. Rosner, "ERK7 is an autoactivated member of the MAPK family," Journal of Biological Chemistry, vol. 276, no. 24, pp. 21272-21279, 2001.

[32] M. K. Abe, W. L. Kuo, M. B. Hershenson, and M. R. Rosner, "Extracellular signal-regulated kinase 7 (ERK7), a novel ERK with a C-terminal domain that regulates its activity, its cellular localization, and cell growth," Molecular and Cellular Biology, vol. 19, no. 2, pp. 1301-1312, 1999.

[33] J. D. Thompson, T. J. Gibson, and D. G. Higgins, "Multiple sequence alignment using ClustalW and ClustalX," Current Protocols in Bioinformatics, vol. 2, unit 2.3, 2002.

[34] D. Sheikh-Hamad, J. Di Mari, W. N. Suki, R. Safirstein, B. A. Watts III, and D. Rouse, "p38 Kinase activity is essential for osmotic induction of mRNAs for HSP70 and transporter for organic solute betaine in Madin-Darby canine kidney cells," Journal of Biological Chemistry, vol. 273, no. 3, pp. 1832-1837, 1998.
[35] Z. S. Han, H. Enslen, X. Hu et al., "A conserved p38 mitogenactivated protein kinase pathway regulates Drosophila immunity gene expression," Molecular and Cellular Biology, vol. 18, no. 6, pp. 3527-3539, 1998.

[36] H. Inoue, N. Hisamoto, H. A. Jae et al., "The C. elegans p38 MAPK pathway regulates nuclear localization of the transcription factor SKN-1 in oxidative stress response," Genes and Development, vol. 19, no. 19, pp. 2278-2283, 2005.

[37] S. A. Wadsworth, D. E. Cavender, S. A. Beers et al., "RWJ 67657 , a potent, orally active inhibitor of p38 mitogenactivated protein kinase," Journal of Pharmacology and Experimental Therapeutics, vol. 291, no. 2, pp. 680-687, 1999.

[38] S. M. Sweitzer, S. Medicherla, R. Almirez et al., "Antinociceptive action of a p38 $\alpha$ MAPK inhibitor, SD-282, in a diabetic neuropathy model," Pain, vol. 109, no. 3, pp. 409-419, 2004.

[39] L. Tong, S. Pav, D. M. White et al., "A highly specific inhibitor of human p38 MAP kinase binds in the ATP pocket," Nature Structural Biology, vol. 4, no. 4, pp. 311-316, 1997.

[40] Z. Wang, B. J. Canagarajah, J. C. Boehm et al., "Structural basis of inhibitor selectivity in MAP kinases," Structure, vol. 6, no. 9, pp. 1117-1128, 1998.

[41] S. Wei, F. Marches, B. Daniel, S. Sonda, K. Heidenreich, and T. Curiel, "Pyridinylimidazole p38 mitogen-activated protein kinase inhibitors block intracellular Toxoplasma gondii replication," International Journal for Parasitology, vol. 32, no. 8, pp. 969-977, 2002.

[42] S. Wei, B. J. Daniel, M. J. Brumlik et al., "Drugs designed to inhibit human p38 mitogen-activated protein kinase activation treat Toxoplasma gondii and Encephalitozoon cuniculi infection," Antimicrobial Agents and Chemotherapy, vol. 51, no. 12, pp. 4324-4328, 2007.

[43] C. M. Doerig, D. Parzy, G. Langsley, P. Horrocks, R. Carter, and C. D. Doerig, "A MAP kinase homologue from the human malaria parasite, Plasmodium falciparum," Gene, vol. 177, no. 1-2, pp. 1-6, 1996.

[44] D. Dorin, P. Alano, I. Boccaccio et al., "An atypical mitogenactivated protein kinase (MAPK) homologue expressed in gametocytes of the human malaria parasite Plasmodium falciparum. Identification of a MAPK signature," Journal of Biological Chemistry, vol. 274, no. 42, pp. 29912-29920, 1999.

[45] D. Dorin, J. P. Semblat, P. Poullet et al., "PfPK7, an atypical MEK-related protein kinase, reflects the absence of classical three-component MAPK pathways in the human malaria parasite Plasmodium falciparum," Molecular Microbiology, vol. 55, no. 1, pp. 184-196, 2005.

[46] P. Ward, L. Equinet, J. Packer, and C. Doerig, "Protein kinases of the human malaria parasite Plasmodium falciparum: the kinome of a divergent eukaryote," BMC Genomics, vol. 5, no. 1, article 79, 2004.

[47] D. Dorin, K. L. Roch, P. Sallicandro et al., "Pfnek-1, a NIMArelated kinase from the human malaria parasite Plasmodium falciparum: biochemical properties and possible involvement in MAPK regulation," European Journal of Biochemistry, vol. 268, no. 9, pp. 2600-2608, 2001.

[48] D. Dorin-Semblat, N. Quashie, J. Halbert et al., "Functional characterization of both MAP kinases of the human malaria parasite Plasmodium falciparum by reverse genetics," Molecular Microbiology, vol. 65, no. 5, pp. 1170-1180, 2007.

[49] J. G. Ellis, M. Davila, and R. Chakrabarti, "Potential involvement of extracellular signal-regulated kinase 1 and 2 in encystation of a primitive eukaryote, Giardia lamblia: stagespecific activation and intracellular localization," Journal of Biological Chemistry, vol. 278, no. 3, pp. 1936-1945, 2003. 
[50] M. J. Brumlik, S. Wei, K. Finstad et al., "Identification of a novel mitogen-activated protein kinase in Toxoplasma gondii," International Journal for Parasitology, vol. 34, no. 11, pp. 1245 1254, 2004.

[51] M. Parsons, E. A. Worthey, P. N. Ward, and J. C. Mottram, "Comparative analysis of the kinomes of three pathogenic trypanosomatids: Leishmania major, Trypanosoma brucei and Trypanosoma cruzi," BMC Genomics, vol. 6, article 127, 2005.

[52] M. Wiese, "Leishmania MAP kinases_familiar proteins in an unusual context," International Journal for Parasitology, vol. 37, no. 10, pp. 1053-1062, 2007.

[53] M. Wiese, "A mitogen-activated protein (MAP) kinase homologue of Leishmania mexicana is essential for parasite survival in the infected host," The EMBO Journal, vol. 17, no. 9, pp. 2619-2628, 1998.

[54] S. B. Hua and C. C. Wang, "Differential accumulation of a protein kinase homolog in Trypanosoma brucei," Journal of Cellular Biochemistry, vol. 54, no. 1, pp. 20-31, 1994.

[55] S. B. Hua and C. C. Wang, "Interferon- $\gamma$ activation of a mitogen-activated protein kinase, KFR1, in the bloodstream form of Trypanosoma brucei," Journal of Biological Chemistry, vol. 272, no. 16, pp. 10797-10803, 1997.

[56] M. Erdmann, A. Scholz, I. M. Melzer, C. Schmetz, and M. Wiese, "Interacting protein kinases involved in the regulation of flagellar length," Molecular Biology of the Cell, vol. 17, no. 4, pp. 2035-2045, 2006.

[57] M. Erdmann, LmxMPK3, a mitogen-activated protein kinase involved in length control of a eukaryotic flagellum, Thesis dissertation, University of Hamburg, Hamburg, Germany, 2009.

[58] Q. Wang, I. M. Melzer, M. Kruse, C. Sander-Juelch, and M. Wiese, "LmxMPK4, a mitogen-activated protein (MAP) kinase homologue essential for promastigotes and amastigotes of Leishmania mexicana," Kinetoplastid Biology and Disease, vol. 4, article 6, 2005.

[59] M. A. Morales, O. Renaud, W. Faigle, S. L. Shorte, and G. F. Späth, "Over-expression of Leishmania major MAP kinases reveals stage-specific induction of phosphotransferase activity," International Journal for Parasitology, vol. 37, no. 11, pp. 1187-1199, 2007.

[60] D. D. Pfister, G. Burkard, S. Morand, C. K. Renggli, I. Roditi, and E. Vassella, "A mitogen-activated protein kinase controls differentiation of bloodstream forms of Trypanosoma brucei," Eukaryotic Cell, vol. 5, no. 7, pp. 1126-1135, 2006.

[61] J. Ellis, M. Sarkar, E. Hendriks, and K. Matthews, "A novel ERK-like, CRK-like protein kinase that modulates growth in Trypanosoma brucei via an autoregulatory C-terminal entension," Molecular Microbiology, vol. 53, no. 5, pp. 14871499, 2004.

[62] M. A. Morales, P. Pescher, and G. F. Späth, "Leishmania major MPK7 protein kinase activity inhibits intracellular growth of the pathogenic amastigote stage," Eukaryotic Cell, vol. 9, no. 1, pp. 22-30, 2010.

[63] F. Bengs, A. Scholz, D. Kuhn, and M. Wiese, "LmxMPK9, a mitogen-activated protein kinase homologue affects flagellar length in Leishmania mexicana," Molecular Microbiology, vol. 55, no. 5, pp. 1606-1615, 2005.

[64] S. John von Freyend, H. Rosenqvist, A. Fink et al., "LmxMPK4, an essential mitogen-activated protein kinase of Leishmania mexicana is phosphorylated and activated by the STE7like protein kinase LmxMKK5," International Journal for Parasitology, vol. 40, no. 8, pp. 969-978, 2010.
[65] D. Kuhn and M. Wiese, "LmxPK4, a mitogen-activated protein kinase kinase homologue of Leishmania mexicana with a potential role in parasite differentiation," Molecular Microbiology, vol. 56, no. 5, pp. 1169-1182, 2005.

[66] B. Rotureau, M. A. Morales, P. Bastin, and G. F. Späth, "The flagellum-mitogen-activated protein kinase connection in Trypanosomatids: a key sensory role in parasite signalling and development?" Cellular Microbiology, vol. 11, no. 5, pp. 710-718, 2009.

[67] S. A. Berman, N. F. Wilson, N. A. Haas, and P. A. Lefebvre, "A novel MAP kinase regulates flagellar length in Chlamydomonas," Current Biology, vol. 13, no. 13, pp. 1145-1149, 2003.

[68] M. Wiese, D. Kuhn, and C. G. Grünfelder, "Protein kinase involved in flagellar-length control," Eukaryotic Cell, vol. 2, no. 4, pp. 769-777, 2003.

[69] I. B. Müller, D. Domenicali-Pfister, I. Roditi, and E. Vassella, "Stage-specific requirement of a mitogen-activated protein kinase by Trypanosoma brucei," Molecular Biology of the Cell, vol. 13, no. 11, pp. 3787-3799, 2002.

[70] J. M. Carlton, R. P. Hirt, J. C. Silva et al., "Draft genome sequence of the sexually transmitted pathogen Trichomonas vaginalis," Science, vol. 315, no. 5809, pp. 207-212, 2007.

[71] D. Ray, S. Dutta, S. Banerjee, R. Banerjee, and S. Raha, "Identification, structure, and phylogenetic relationships of a mitogen-activated protein kinase homologue from the parasitic protist Entamoeba histolytica," Gene, vol. 346, pp. 41$50,2005$.

[72] C. Naula, M. Parsons, and J. C. Mottram, "Protein kinases as drug targets in trypanosomes and Leishmania," Biochimica et Biophysica Acta, vol. 1754, no. 1-2, pp. 151-159, 2005.

[73] B. Ge, H. Gram, F. Di Padova et al., "MAPKK-independent activation of $\mathrm{p} 38 \alpha$ mediated by TAB1-dependent autophosphorylation of p38 $\alpha$," Science, vol. 295, no. 5558, pp. 12911294, 2002.

[74] T. Tanoue, M. Adachi, T. Moriguchi, and E. Nishida, "A conserved docking motif in MAP kinases common to substrates, activators and regulators," Nature Cell Biology, vol. 2, no. 2, pp. 110-116, 2000.

[75] T. Tanoue, R. Maeda, M. Adachi, and E. Nishida, "Identification of a docking groove on ERK and p38 MAP kinases that regulates the specificity of docking interactions," The EMBO Journal, vol. 20, no. 3, pp. 466-479, 2001.

[76] M. Awale, V. Kumar, P. Saravanan, and C. G. Mohan, "Homology modeling and atomic level binding study of Leishmania MAPK with inhibitors," Journal of Molecular Modeling, vol. 16, no. 3, pp. 475-488, 2010.

[77] K. P. Wilson, M. J. Fitzgibbon, P. R. Caron et al., "Crystal structure of p38 mitogen-activated protein kinase," Journal of Biological Chemistry, vol. 271, no. 44, pp. 27696-27700, 1996.

[78] M. Huse and J. Kuriyan, "The conformational plasticity of protein kinases," Cell, vol. 109, no. 3, pp. 275-282, 2002.

[79] L. Kim, L. Del Rio, B. A. Butcher et al., "p38 MAPK autophosphorylation drives macrophage IL-12 production during intracellular infection," Journal of Immunology, vol. 174, no. 7, pp. 4178-4184, 2005.

[80] R. Rangarajan, A. K. Bei, D. Jethwaney et al., "A mitogenactivated protein kinase regulates male gametogenesis and transmission of the malaria parasite Plasmodium berghei," EMBO Reports, vol. 6, no. 5, pp. 464-469, 2005.

[81] R. Tewari, D. Dorin, R. Moon, C. Doerig, and O. Billker, "An atypical mitogen-activated protein kinase controls cytokinesis 
and flagellar motility during male gamete formation in a malaria parasite," Molecular Microbiology, vol. 58, no. 5, pp. 1253-1263, 2005.

[82] A. R. Renslo and J. H. McKerrow, "Drug discovery and development for neglected parasitic diseases," Nature Chemical Biology, vol. 2, no. 12, pp. 701-710, 2006. 

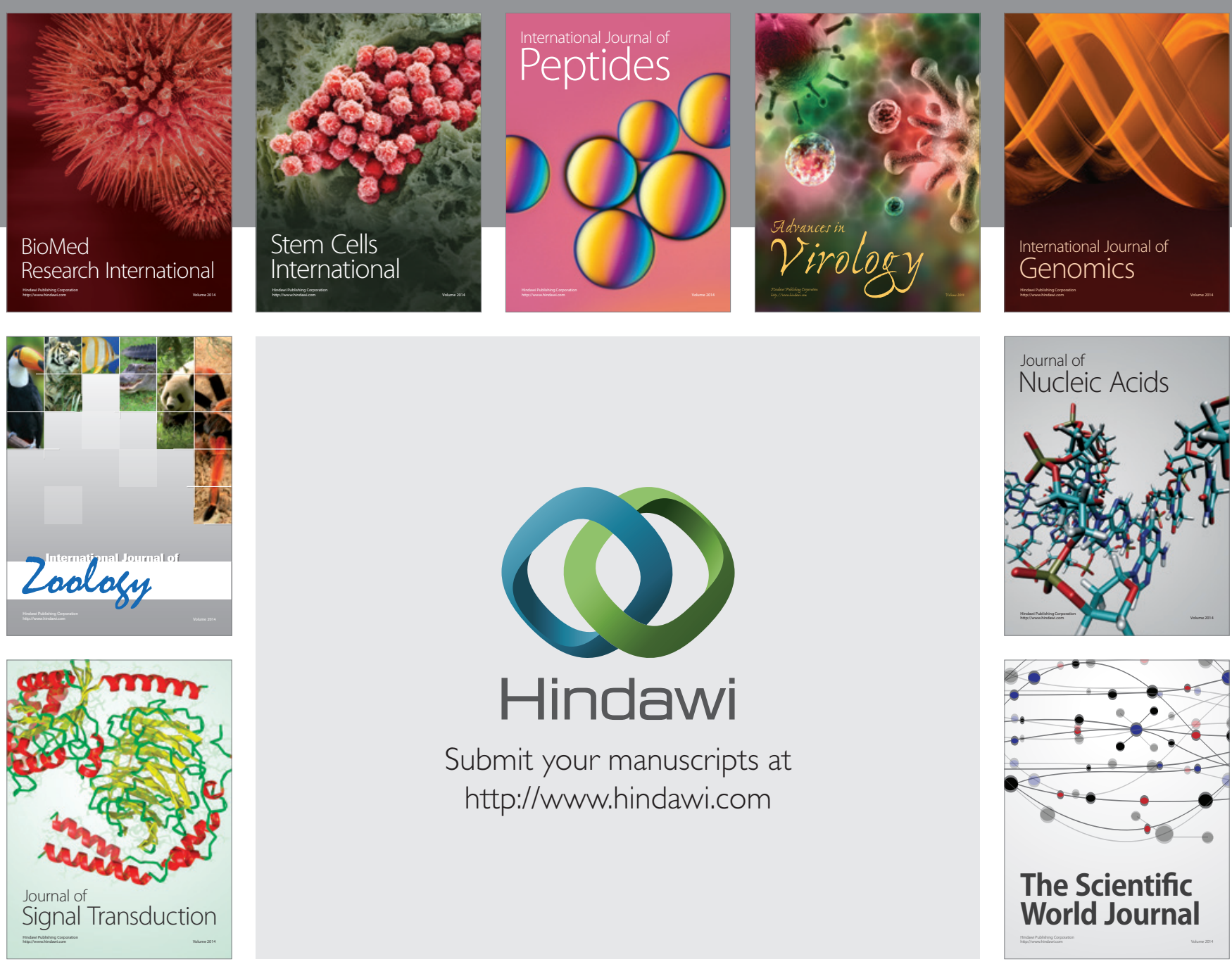

Submit your manuscripts at

http://www.hindawi.com
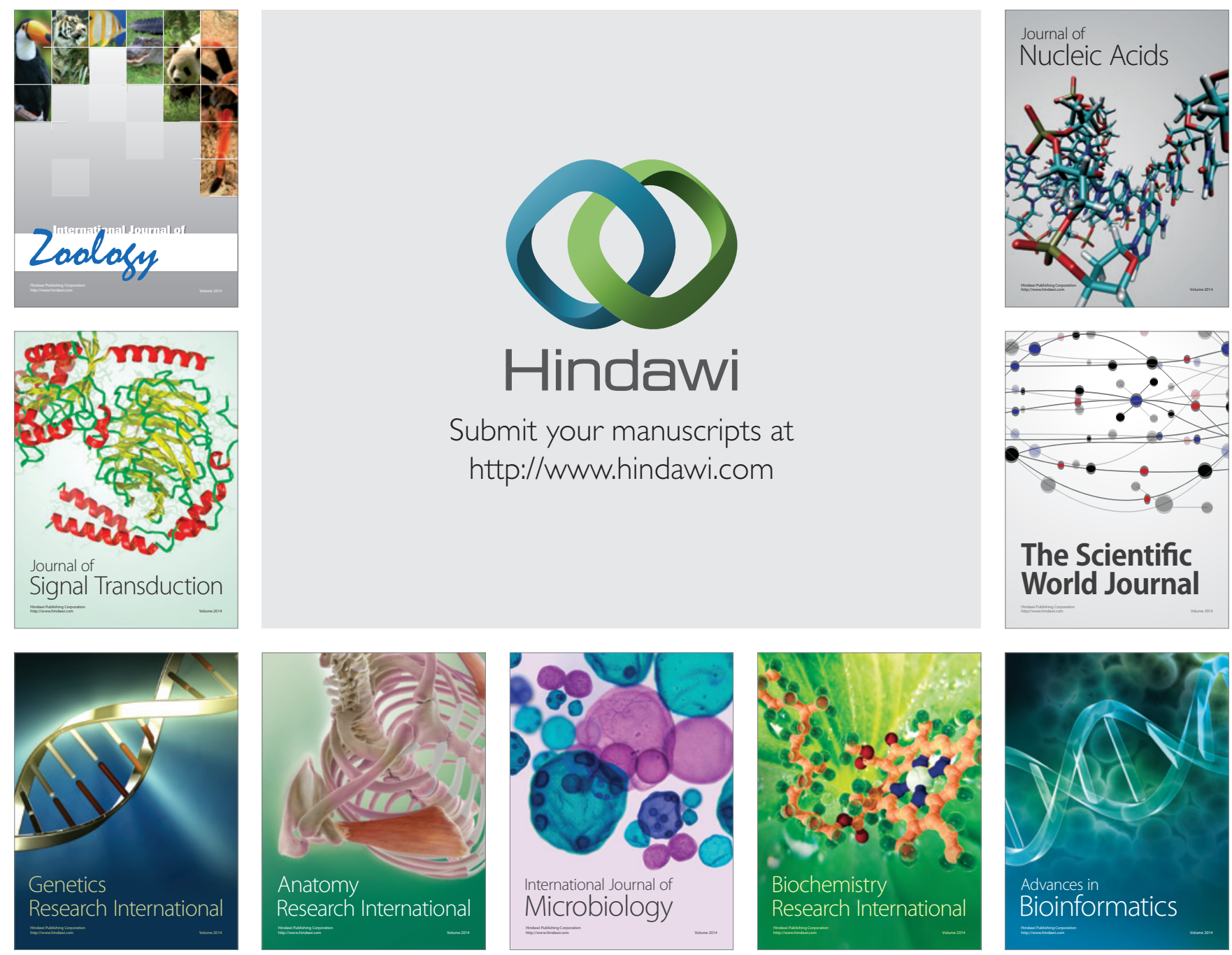

The Scientific World Journal
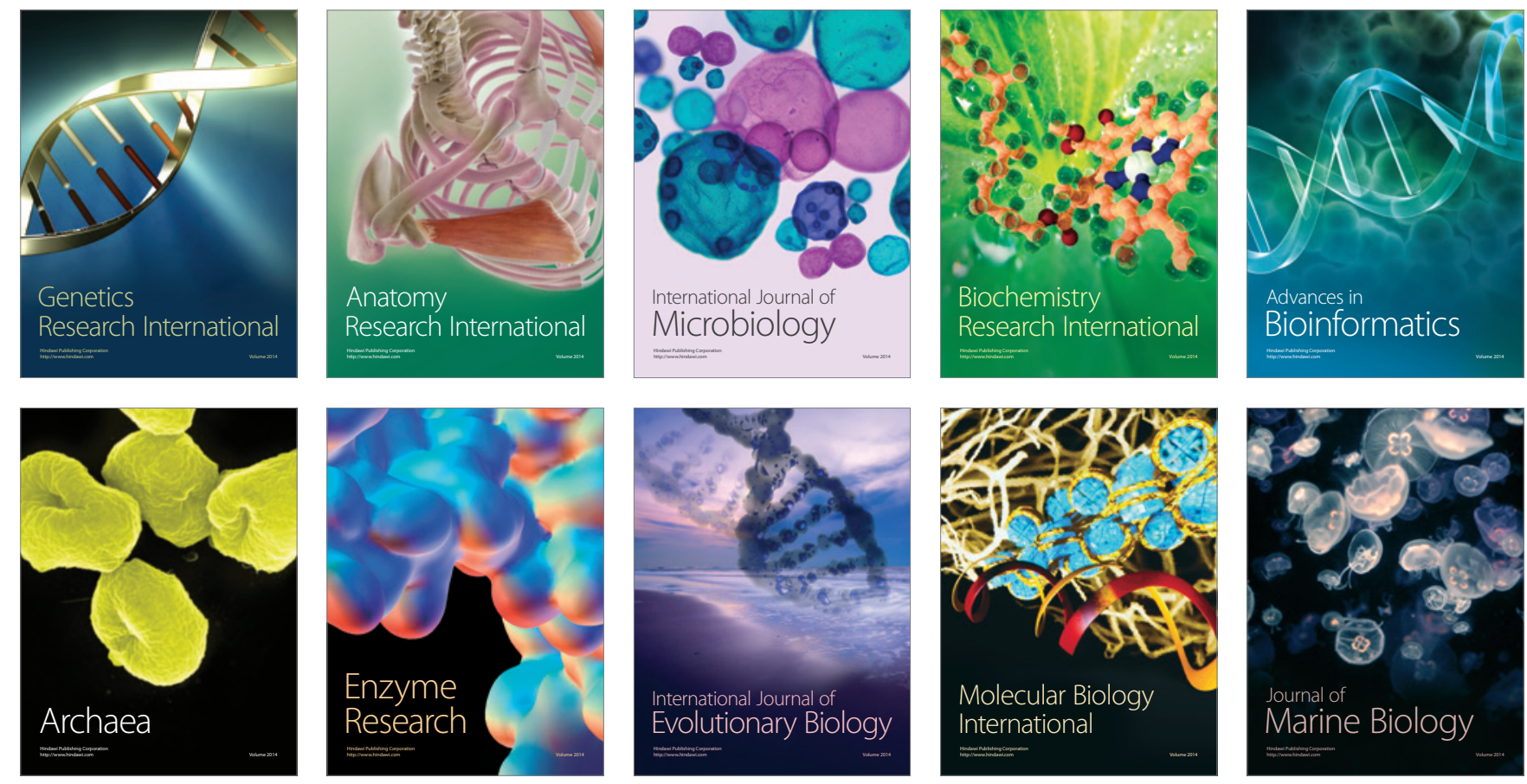